\title{
A UNIQUENESS THEOREM FOR PROPERLY EMBEDDED MINIMAL SURFACES BOUNDED BY STRAIGHT LINES
}

\author{
FRANCISCO J. LOPEZ and FRANCISCO MARTIN
}

(Received 22 May 1999; revised 5 April 2000)

Communicated by K. Ecker

\begin{abstract}
In this paper we prove a uniqueness theorem for minimal discs in $\mathbb{R}^{3}$ spanning a polygonal boundary. 2000 Mathematics subject classification: primary 53A10; secondary 53C42.

Keywords and phrases: properly embedded minimal surfaces, boundary, straight lines.
\end{abstract}

\section{Introduction}

A classical problem considered by Schwarz, Weierstrass and Riemann was to determine minimal surfaces bounded by straight lines. These authors obtained existence results for minimal surfaces with boundary a given polygon, where the sides of the polygon could be of finite or infinite length.

Jenkins and Serrin [6] proved an existence and uniqueness theorem for minimal graphs bounded by straight lines. They obtained simple, necessary and sufficient conditions to solve the Dirichlet problem in a compact convex domain bounded by a polygon assuming values $+\infty,-\infty$ and continuous data on different straight segments in the boundary.

In [9], the authors construct a deformation of some particular Jenkins-Serrin graphs (see Figure 1) which consists of properly embedded minimal discs bounded by straight lines, contained in a wedge of a slab (see Figure 3). These examples arise as a solution to Plateau's problem for a polygonal non-compact boundary consisting of a twice $\mathbf{U}$ shaped contour. These surfaces can be used as a new type of barrier for the maximum principle application. As a consequence, in [9], the authors have proved that any

Research partially supported by DGICYT grant number PB97-0785.

(C) 2000 Australian Mathematical Society 0263-6115/2000\$A2.00+0.00 


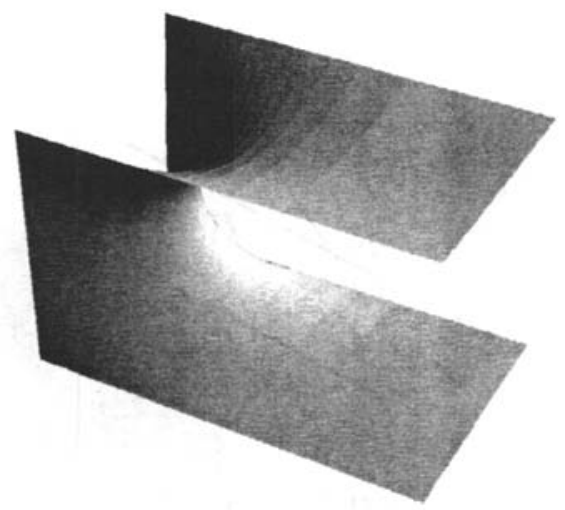

FIGURE 1. A Jenkins-Serrin graph.

properly immersed minimal surface in a slab wedge of angle less than $\pi$ lies in the convex hull of its boundary. Furthermore, they have proved non existence results for minimal surfaces with planar boundaries which generalize some classical results by Nitsche [12] (see also Rossman's work [15] for a good survey).

One of the most interesting questions in regards to Plateau's problem is to determine how many minimal surfaces can be spanned in a given boundary. The answer to this question is not generally known, not even if we fix the topological type of the solutions. The difficulty increases if, in addition, the boundary is not compact.

An existence and uniqueness theorem for properly immersed minimal discs whose boundaries consist of two disjoint straight lines and a segment which joins them orthogonally has been recently obtained in [10]. In [4], Fang have considered the non-compact Plateau's problem for surfaces of annular type bounded by a pair of non-compact convex curves in parallel planes, obtaining existence results for certain symmetric boundaries.

The aim of this paper is to obtain a uniqueness theorem for the surfaces in the family of examples exhibited in [9]. To be more precise, we deal with the uniqueness of properly embedded minimal surfaces whose boundary $\Gamma_{\theta d}$ consists of the following configuration of straight lines:

Fix $\theta \in\left[0, \pi\left[\right.\right.$ and $d \geq 0$, and consider two half-lines $r_{1}^{+}$and $r_{1}^{-}$in $\mathbb{R}^{3}$, meeting at an angle of $\theta$. If $\theta=0$ this means that the straight lines are parallel and distinct. Let $q_{1}^{+}$and $q_{1}^{-}$be two points in $r_{1}^{+}$and $r_{1}^{-}$, respectively, such that they are symmetric with respect to the inner bisector of these half-lines. If and only if $\theta \neq 0$, we allow $q_{1}^{+}=q_{1}^{-}$. We choose $q_{1}^{+}$and $q_{1}^{-}$in such a way that either $q_{1}^{+}=q_{1}^{-}$or the half-lines $\ell_{1}^{+}$and $\ell_{1}^{-}$on $r_{1}^{+}$and $r_{1}^{-}$starting at $q_{1}^{+}$and $q_{1}^{-}$, respectively, do not intersect. Write $d=\operatorname{dist}\left(q_{1}^{+}, q_{1}^{-}\right)$.

Let $\pi_{1}$ be the plane determined by $\ell_{1}^{+}$and $\ell_{1}^{-}$and let $\pi_{2}$ denote a plane parallel 


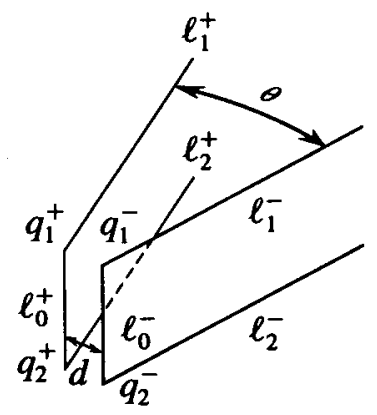

FIGURE 2. The curve $\Gamma_{\theta d}$.

and distinct to $\pi_{1}$. Let $\ell_{2}^{+}$and $\ell_{2}^{-}$be the orthogonal projections to $\pi_{2}$ of $\ell_{1}^{+}$and $\ell_{1}^{-}$, respectively. Denote by $q_{2}^{+}\left(q_{2}^{-}\right)$the orthogonal projection to $\pi_{2}$ of $q_{1}^{+}\left(q_{1}^{-}\right)$, and label by $\ell_{0}^{+}\left(\ell_{0}^{-}\right)$the segment $\left[q_{1}^{+}, q_{2}^{+}\right]\left(\left[q_{1}^{-}, q_{2}^{-}\right]\right)$. Finally, we write

$$
\Gamma_{\theta d}^{+}=\bigcup_{i=0}^{2}\left(\ell_{i}^{+}\right), \quad \Gamma_{\theta d}^{-}=\bigcup_{i=0}^{2}\left(\ell_{i}^{-}\right)
$$

and define

$$
\Gamma_{\theta d}=\Gamma_{\theta d}^{+} \cup \Gamma_{\theta d}^{-} .
$$

We consider the following generalized Plateau's problem:

PROBLEM. Determine a properly immersed minimal surface $X: M \rightarrow \mathbb{R}^{3}$ satisfying:

(1) $M$ is homeomorphic to the closed unit disc $\overline{\mathbb{D}}$ minus two boundary points $E_{1}$ and $E_{2}$, that we call the ends of $M$.

(2) $X(\partial(M))=\Gamma_{\theta d}$.

(3) If $d>0, X$ is an embedding.

(4) In the limit case $\ell_{0}^{+}=\ell_{0}^{-}$(that is, $d=0$ ), the maps $\left.X\right|_{M-\gamma^{+}}$and $\left.X\right|_{M-\gamma^{-}}$are injective, where $\gamma^{+}$and $\gamma^{-}$are the two connected components of $\partial(M)$.

(5) $X(M)$ lies in the convex hull, $\mathscr{E}\left(\Gamma_{\theta d}\right)$, of $\Gamma_{\theta d}$.

In the limit case $\theta=0$, it is known that $0<d<\|\vec{v}\|$, where $\vec{v}$ is the vector with origin $q_{1}^{+}$and end $q_{2}^{+}$, and the example is uniquely determined as one of the above mentioned Jenkins-Serrin graphs. See the remark on page 370.

Concerning to the general case $\theta>0$, we have proved the following:

MAIN THEOREM. There exists $d_{\theta}>0$ such that: 


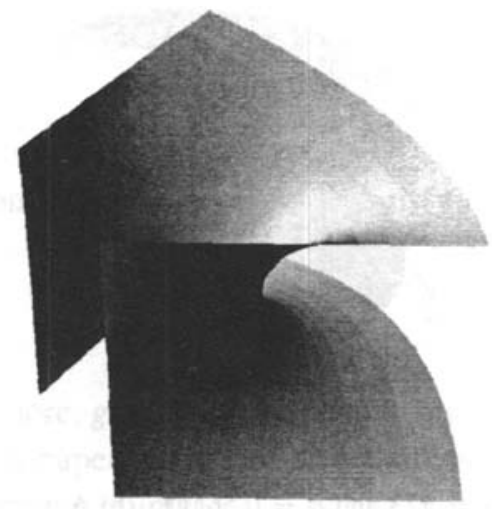

(a)

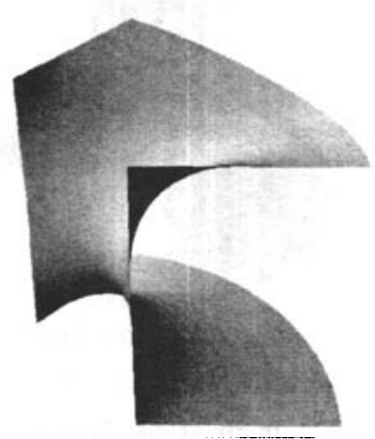

(b)

FIGURE 3. The two solutions in case $\theta=\pi / 3, d=0.56\|\vec{v}\|$. Figure (a) corresponds to an unstable example, and Figure (b) corresponds to a stable one.

(A) - If $d \in] 0, d_{\theta}[$, the above Plateau's problem has only two solutions.

- If $d=0$ or $d=d_{\theta}$, the above Plateau's problem has a unique solution.

(B) For $d>d_{\theta}$, there are no solutions.

As we mentioned above, the existence part of this result can be found in [9]. Anyway, in Section 2 we briefly describe this family of surfaces. The statement of this theorem also involves the uniqueness of the solutions, which is the subject of this paper. This result is an elaborate consequence of Theorem 3.8, Theorem 3.12, Theorem 3.17, Theorem 3.24 and Theorem 3.32 in this paper.

Finally, note that the above assumption (5) cannot be derived from the ones described in (1)-(4). As a matter of fact, in Figure 4 we illustrate two examples satisfying (1), (2), (3) and (4), but none of them is contained in the convex-hull of its boundary.

This paper is organized as follows. In Section 2, we give several results we need in this paper. We also describe the family of minimal surfaces arising as solutions of Plateau's problem above, and recall its geometrical properties. In Section 3, we obtain the main uniqueness results for the space of solutions. In Subsection 3.1 we prove that $M$ is conformally equivalent to a twice punctured closed disc with piecewise analytic boundary (see Paragraph 3.1.1), and in Paragraph 3.1.2 we prove that the meromorphic data of $X$ extend to the closed disc. In the case $\theta / \pi \in \mathbb{Q}$, it is not hard to see that these results are a consequence of the Cone Lemma, proved by Hoffman and Meeks in [5]. Our approach also satisfactorily answers the case $\theta / \pi \notin \mathbb{Q}$. In Subsection 3.2, we prove that any solution of Plateau's problem above inherits the symmetries of its boundary. This fact is a consequence of the reflection method of 


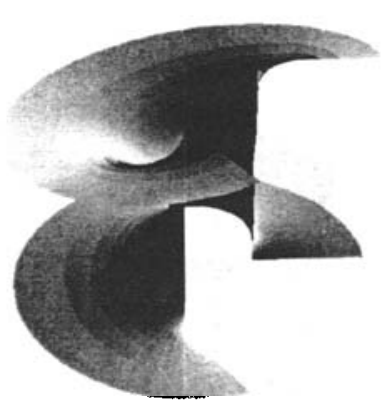

(a)

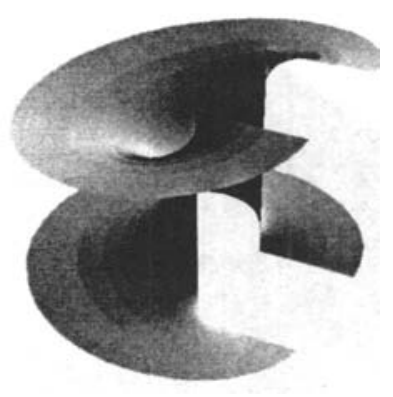

(b)

FIGURE 4. (a) A minimal disc bounded by $\Gamma_{\theta d}$, for $\theta=\pi / 3$ and $d=0.56\|\vec{v}\|$. (b) A minimal disc bounded by $\Gamma_{\theta d}$, for $\theta=0$ and $d=0.40\|\vec{v}\|$.

Alexandrov. In this sense, we generalize the ideas of Schoen to our particular case of non-compact boundary with unbounded slope. Finally, in Subsection 3.3 we make use of the results in the preceding subsections to determine a model of the complex structure and Weierstrass representation of any solution of our Plateau problem.

\section{Background and notation}

The aim of this section is to fix the principal notation used in this paper, and to summarize some results about complete minimal surfaces.

Let $X: M \rightarrow \mathbb{R}^{3}$ be a proper conformal minimal immersion, where $M$ is a Riemann surface with piecewise analytic boundary homeomorphic to the closed unit disc $\mathbb{D}$ minus two boundary points $E_{1}$ and $E_{2}$, that we call the ends of $M$.

REMARK. We say that $M$ is a Riemann surface with piecewise analytic boundary if and only if $M$ is a subset of an open Riemann surface $M^{\prime}$, the conformal structure of $M-\partial(M)$ is that induced by $M^{\prime}$ and $\partial(M)$ consists of a set of piecewise analytic curves. Meromorphic (respectively holomorphic) functions and 1-forms on $M$ are, by definition, the restriction of meromorphic (respectively holomorphic) functions and 1 -forms on $M^{\prime}$.

The Weierstrass representation of $X$ is denoted by $(g, \eta)$. Recall that $g$ is a meromorphic function and $\eta$ a holomorphic 1-form on $M$. Both of them determine the minimal immersion $X$ as follows:

$$
X(P)=\operatorname{Re}\left(\int^{P}\left(\phi_{1}, \phi_{2}, \phi_{3}\right)\right),
$$


where

$$
\phi_{1}=\frac{1}{2}\left(1-g^{2}\right) \eta, \quad \phi_{2}=\frac{i}{2}\left(1+g^{2}\right) \eta, \quad \phi_{3}=g \eta
$$

are holomorphic 1-forms on $M$ satisfying

$$
\sum_{j=1}^{3}\left|\phi_{j}\right|^{2} \neq 0
$$

Furthermore, $g$ is the stereographic projection of the Gauss map $N: M \rightarrow \mathbb{S}^{2}$.

In this paper we often use the following classical results about minimal surfaces with common points.

Let $M_{1}$ and $M_{2}$ be two minimal surfaces in $\mathbb{R}^{3}$. Assume $p_{0}$ is an interior point of both $M_{1}$ and $M_{2}$ and suppose $T_{p_{0}} M_{1}=T_{p_{0}} M_{2}$. Up to a rigid motion, assume that $p_{0}=(0,0,0)$ and $T_{p_{0}} M_{1}=\left\{x_{3}=0\right\}$, so that both $M_{1}$ and $M_{2}$ are given near $p_{0}$ as the graphs of two real analytic functions $u_{1}\left(x_{1}, x_{2}\right)$ and $u_{2}\left(x_{1}, x_{2}\right)$, respectively.

The difference $u\left(x_{1}, x_{2}\right)=u_{1}\left(x_{1}, x_{2}\right)-u_{2}\left(x_{1}, x_{2}\right)$ can be expanded as a power series $u\left(x_{1}, x_{2}\right)=\sum_{k=1}^{\infty} f_{k}\left(x_{1}, x_{2}\right)$ in a neighbourhood of the origin, where each $f_{k}\left(x_{1}, x_{2}\right)$ is a homogeneous polynomial of degree $k$ in $x_{1}$ and $x_{2}$. If $f_{k}\left(x_{1}, x_{2}\right) \equiv 0,1 \leq k \leq v-1$, and $f_{v}\left(x_{1}, x_{2}\right)$ does not vanish identically, then we say that $M_{1}$ and $M_{2}$ have a contact of $(v-1)$ th order at $p_{0}$. If $v-1=1$, we say that they have an ordinary contact. In case $v=1$, the surfaces are transverse at $p_{0}$.

THEOREM 2.1 (Order of contact). Two minimal surfaces $M_{1}$ and $M_{2}$ have a contact of order $v-1$ at $p_{0}$ if and only if $M_{1}$ intersects $M_{2}$ along $v$ curves $\mathscr{C}_{1}, \ldots, \mathscr{C}_{v}$ in a neighbourhood of $p_{0}$. If $v-1>0$, these curves intersect each other at $p_{0}$ at an angle $\pi / \nu$. They divide a neighbourhood of $p_{0}$ in $M_{1}$ into $2 v$ open sectors, such that $M_{1}$ lies on one side of $M_{2}$ in one sector and on the other side in the next sector.

Furthermore, if $m_{i}$ is the multiplicity of the Gauss map of $M_{i}$ at $p_{0}, i=1,2$, then

$$
v-1 \geq \operatorname{Minimum}\left\{m_{1}, m_{2}\right\} \text {. }
$$

In particular, when $M_{2}$ is the tangent plane $T_{p_{0}} M_{1}$ at $p_{0}$, the multiplicity of the Gauss map $g$ of $M_{1}$ at $p_{0}$ is $v-1$ if, and only if, $T_{p_{0}} M_{1}$ intersects the surface along $v$ curves $\mathscr{C}_{1}, \ldots, \mathscr{C}_{v}$ in a neighbourhood of $p_{0}$. As above, these curves intersect each other at $p_{0}$ forming angles different to 0 and $\pi$. They divide a neighbourhood of $p_{0}$ into $2 v$ open sectors, such that $M_{1}$ lies on one side of the tangent plane in one sector and on the other side in the next sector.

We say that $p_{0}$ is an ordinary point of contact if the multiplicity of the Gauss map at $p_{0}$ is 1 . Obviously, at an ordinary point of contact the tangent plane to a minimal surface intersects the surface along two orthogonal curves. 
Minimal surfaces containing straight lines have special properties. These were studied by Schwarz, who obtained the following result:

THEOREM 2.2 (Schwarz's Reflection Principle). If a minimal surface contains a piece of a straight line $\ell$ in its boundary, then the union of the surface and its 180 degrees rotation about $\ell$ is a minimal surface which has $\ell$ as an axis of symmetry.

Two good references about these results are [13, Section 437] and [3, Section 3.4].

Subsection 3.2 is devoted to proving that the minimal surface $X(M)$ inherits the symmetries of its boundary. The proof of these facts is a suitable version of the reflection method of Alexandrov [1] and, as such, is based essentially on the Hopf maximum principle. We state two theorems which summarize the well-known versions of the maximum principle which we require.

THEOREM 2.3 (Interior maximum principle). Suppose $M_{1}, M_{2}$ are minimal surfaces in $\mathbb{R}^{3}$. Suppose $p$ is an interior point of both $M_{1}$ and $M_{2}$, and suppose $T_{p} M_{1}=T_{p} M_{2}$. Assume that $T_{p} M_{1}=\left\{x_{3}=0\right\}$ so that both $M_{1}, M_{2}$ are given near $p$ as the graphs of two real analytic functions $u_{1}$ and $u_{2}$, respectively. If $u_{1} \geq u_{2}$ in a neighbourhood of $p$, then $M_{1}=M_{2}$.

An elementary consequence of this result is the non-existence of compact nonplanar minimal surfaces with boundary contained in a plane $\Pi$. This result remains true in the non-compact case if in addition the surface lies in the slab determined by $\Pi$ and a plane $\Pi^{\prime}$ parallel to $\Pi$. See [11, Lemma 2.1] for details.

THEOREM 2.4 (Maximum principle at the boundary). Suppose $M_{1}, M_{2}$ are minimal surfaces in $\mathbb{R}^{3}$ with boundaries $\beta_{1}$ and $\beta_{2}$. Suppose $p$ is an interior point of both $\beta_{1}$ and $\beta_{2}$, and suppose $T_{p} M_{1}=T_{p} M_{2}, T_{p} \beta_{1}=T_{p} \beta_{2}$. Assume that $T_{p} M_{1}=\left\{x_{3}=0\right\}$ so that both $M_{1}, M_{2}$ are given near $p$ as the graphs of two real analytic functions $u_{1}$ and $u_{2}$, respectively. If $u_{1} \geq u_{2}$ in a neighbourhood of $p$, then $M_{1}=M_{2}$.

We conclude these preliminaries describing the family of minimal surfaces constructed by the authors in [9]. We also include a summary of the main geometrical properties of these examples.

Take $n \in[1,2]$ and $r \in]-1,1\left[\right.$. Put $\left.r=-\cos \left(x_{0}\right), x_{0} \in\right] 0, \pi[$. Consider the Riemann surface

$$
\mathscr{N}=\left\{(z, w) \in \mathbb{C}^{*} \times \mathbb{C} / w^{2}=\left(z-e^{i x_{0} / n}\right)\left(z-e^{-i x_{0} / n}\right)\right\}
$$




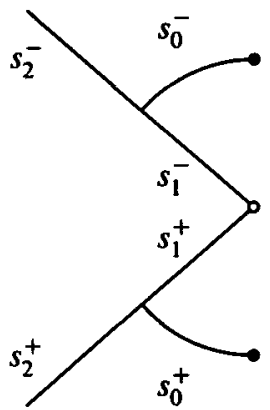

(a)

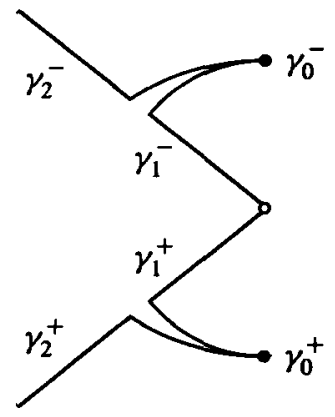

(b)

FIGURE 5. (a) The domain $z(M)$. (b) The surface $M$.

and define in the $z$-plane:

$$
\begin{array}{ll}
\left.\left.s_{1}^{+}=\left\{\lambda e^{-\pi i / n}: \lambda \in\right] 0,1\right]\right\}, & \left.\left.s_{1}^{-}=\left\{\lambda e^{\pi i / n}: \lambda \in\right] 0,1\right]\right\}, \\
s_{2}^{+}=\left\{\lambda e^{-\pi i / n}: \lambda \in[1,+\infty[\},\right. & s_{2}^{-}=\left\{\lambda e^{\pi i / n}: \lambda \in[1,+\infty[\},\right. \\
s_{0}^{+}=\left\{e^{-i t}: t \in\left[x_{0} / n, \pi / n\right]\right\}, & s_{0}^{-}=\left\{e^{i t}: t \in\left[x_{0} / n, \pi / n\right]\right\} .
\end{array}
$$

Then, label $C \subset \mathscr{N}$ as the connected component of $z^{-1}\left(\mathbb{C}-\left(\bigcup_{i=0}^{2}\left(s_{i}^{+} \cup s_{i}^{-}\right)\right)\right)$ containing the point

$$
P_{0}=\left(1,+\sqrt{2\left(1-\cos \left(x_{0} / n\right)\right)}\right)
$$

Define

$$
M=\bar{C}
$$

where $\bar{C}$ means the closure of $C$ in $\mathscr{N}$.

Finally, label $\gamma_{i}^{+}=z^{-1}\left(s_{i}^{+}\right), \gamma_{i}^{-}=z^{-1}\left(s_{i}^{-}\right)$for $i=0,1,2$. Denote

$$
\gamma^{+}=\bigcup_{i=0}^{2} \gamma_{i}^{+}, \quad \gamma^{-}=\bigcup_{i=0}^{2} \gamma_{i}^{-} \text {. }
$$

It is clear that $\partial(M)=\gamma^{+} \cup \gamma^{-}$. Furthermore, note that $\left.z\right|_{\gamma_{i}^{+}}$and $\left.z\right|_{\gamma_{i}^{-}}$are bijective maps onto $s_{i}^{+}$and $s_{i}^{-}$, respectively, $i=1,2$. However, $\gamma_{0}^{+}$and $\gamma_{0}^{-}$consist of two copies of $s_{0}^{+}$and $s_{0}^{-}$, respectively. See Figure 5 for more details.

Since $z(M)$ is simply connected and $0 \notin z(M)$, then the function $z^{n}+z^{-n}+2 r$ is well defined on $M$. We choose the branch of $z^{n}$ satisfying $1^{n}=1$. 
This choice of the branch of $z^{n}$ implies that the function $z^{n}+z^{-n}+2 r$ has neither zeroes nor poles on $M-\partial(M)$.

Hence, the function $\varphi((z, w))=\sqrt{z^{n}+z^{-n}+2 r}$ has a well defined branch on the (simply connected) domain $M-\partial(M)$, that can be extended continuously to $M$. For convenience, we choose the branch satisfying $\varphi\left(P_{0}\right)<0$. Moreover, note that given $z_{0} \in\left(s_{0}^{+} \cup s_{0}^{-}\right)$and denoting $\left\{P^{+}, P^{-}\right\}=z^{-1}\left(z_{0}\right)$, we have $\varphi\left(P^{+}\right)=-\varphi\left(P^{-}\right)$.

We consider the meromorphic data on $M$

$$
g=i z, \quad \phi_{3}=B \frac{d z}{z \varphi}
$$

where $B>0$.

For simplicity, we write

$$
\tau_{r}=\frac{d z}{z \varphi}
$$

and as usual, we denote

$$
\left(\phi_{1}, \phi_{2}, \phi_{3}\right)=\frac{B}{2}\left(-i(1 / z+z) \tau_{r},(1 / z-z) \tau_{r}, 2 \tau_{r}\right) .
$$

As $M$ is homeomorphic to a closed disc minus two boundary points, then

$$
\begin{gathered}
X: M \rightarrow \mathbb{R}^{3}, \\
X(P)=\operatorname{Re} \int_{P_{0}}^{P}\left(\phi_{1}, \phi_{2}, \phi_{3}\right)
\end{gathered}
$$

is a well defined conformal minimal immersion of $M$ in $\mathbb{R}^{3}$.

REMARK. If $n=2$ and $r \in]-1,1$, the immersions associated to the Weierstrass data (4) are known and correspond to some Jenkins-Serrin graphs (see Figure 1). Following the notation of [6], these minimal graphs are the only ones with boundary values $+\infty, 0,+\infty, 0$ on a rectangle. See Karcher's work [7] for a good setting.

For the reasons explained in the last remark, we are going to restrict our attention to the case $n<2$, and in what follows we suppose $n \in[1,2[$.

Concerning to its symmetries, let $S_{h}, S_{v}$ denote the antiholomorphic transformations on $M$

$$
S_{h}((z, w))=(1 / \bar{z}, \bar{w} / \bar{z}), \quad S_{v}((z, w))=(\bar{z}, \bar{w}) .
$$

Notice that $S_{h}\left(P_{0}\right)=S_{v}\left(P_{0}\right)$, and

$$
\begin{array}{ll}
g \circ S_{h}=1 / \bar{g}, & g \circ S_{v}=-\bar{g}, \\
S_{h}^{*}\left(\phi_{3}\right)=-\overline{\phi_{3}}, & S_{v}^{*}\left(\phi_{3}\right)=\overline{\phi_{3}} ;
\end{array}
$$


so elementary arguments imply that $S_{h}$ (respectively $S_{v}$ ) induces on $X(M)$ a symmetry with respect to the plane $x_{3}=0$ (respectively $x_{1}=0$ ).

The following theorem has been proved in [9].

THEOREM 2.5. Let $\theta \in] 0, \pi]$ and $r \in]-1,1[$.

Take $n=2 \pi /(\theta+\pi)$ and consider the Weierstrass data $\left(M, g, \phi_{3}\right)$, where $M$ is described in Figure 5-(b), and

$$
g=i z, \quad \phi_{3}=B \frac{d z}{z \sqrt{z^{n}+z^{-n}+2 r}}, \quad B>0 .
$$

Then, the minimal immersion

$$
X=\operatorname{Re} \int_{1}\left(\frac{1}{2}\left(g^{-1}-g\right) \phi_{3}, \frac{i}{2}\left(g^{-1}+g\right) \phi_{3}, \phi_{3}\right)
$$

satisfies:

(i) $X(M)$ is a properly immersed minimal disk with two boundary ends in $\mathbb{R}^{3}$.

(ii) $X(\partial(M))=\Gamma=\bigcup_{i=0}^{2}\left(\ell_{i}^{+} \cup \ell_{i}^{-}\right)$, where:

(1) The curves $\ell_{1}^{+}$and $\ell_{1}^{-}$are half-lines contained in a plane $x_{3}=k, k>0$, they are symmetric with respect to the plane $x_{1}=0$, and the straight lines containing them meet at an angle $\theta$.

(2) The curve $\ell_{2}^{+}$(respectively $\ell_{2}^{-}$) is the image under the symmetry with respect to the plane $x_{3}=0$ of $\ell_{1}^{+}$(respectively $\ell_{1}^{-}$).

(3) The curve $\ell_{0}^{+}$(respectively $\ell_{0}^{-}$) is the vertical segment joining the end points of $\ell_{1}^{+}$and $\ell_{2}^{+}$(respectively $\ell_{1}^{-}$and $\ell_{2}^{-}$).

(iii) If $\theta \in] 0, \pi[, X(M)$ is contained in the convex-hull $\mathscr{E}(\Gamma)$ of $\Gamma$. If $\theta=\pi$, $X(M)$ is contained in the intersection of the slab $-k \leq x_{3} \leq k$ and one of the two halfspaces determined by the plane containing $\ell_{i}^{+}, \ell_{i}^{-}, i=0,1,2$.

(iv) $X(M)$ is invariant under the symmetries with respect to the planes $x_{1}=0$ and $x_{3}=0$.

(v) Up to a suitable choice of $B$ (rescaling), we can suppose $k=1 / 2$. Then, denote by $d$ oriented distance between $\ell_{0}^{+}$and $\ell_{0}^{-}$. This means that

(1) $|d|=\operatorname{dist}\left(\ell_{0}^{+}, \ell_{0}^{-}\right)$;

(2) $d \leq 0$ if and only if $\ell_{i}^{+} \cap \ell_{i}^{-} \neq \emptyset, i=1,2$, and $d>0$ otherwise;

(3) $d=0$ if and only if $\ell_{0}^{+}=\ell_{0}^{-}$.

Obviously, the function d depends on $r$ and $\theta$. Moreover, for each $\theta \in] 0, \pi]$, there exists a unique $\left.r_{\theta} \in\right] 0,1\left[\right.$ such that $d\left(r_{\theta}\right)=0$. The function $d(r)$ is negative in ] $r_{\theta}, 1[$ and positive in $]-1, r_{\theta}\left[\right.$. Furthermore, $\lim _{r \rightarrow-1} d(r)=0$ and $d(r)$ has an unique critical point $\left.r_{\theta}^{\prime} \in\right]-1, r_{\theta}\left[\right.$. The number $d_{\theta}=d\left(r_{\theta}^{\prime}\right)$ is the maximum of the distance function $d(r)$ on $\left.]-1, r_{\theta}\right]$. 
(vi) $X$ is an embedding if and only if $d>0$. If $d=0$ (that is, $r=r_{\theta}$ ), then $\left.X\right|_{M-\gamma^{+}}$ and $\left.X\right|_{M-\gamma^{-}}$are injective, where $\gamma^{+}$and $\gamma^{-}$are the two connected components of $\partial(M)$.

Up to rescaling, and in what follows, we suppose that $k=1 / 2$, and so, $X(M)$ lies in the slab $-1 / 2 \leq x_{3} \leq 1 / 2$.

The numbers $r \in]-1,1[$ and $\theta \in] 0, \pi]$ are analytical parameters of our family of surfaces. To be more precise and when it is necessary, we can refer to $X_{\theta r}$ as the immersion arising in above theorem for the values $r$ and $\theta$. Analogously, we write $M_{\theta r} \equiv M$ as the underlying complex structure of the disc.

Then we can write the family $\mathscr{M}$ of surfaces arising from Theorem 2.5 as follows:

$$
\left.\left.\mathscr{M}=\left\{X_{\theta r}: M_{\theta r} \rightarrow \mathbb{R}^{3} / r \in\right]-1,1[, \theta \in] 0, \pi\right]\right\} .
$$

Note that $\theta$ has a clear geometrical meaning: the angle that $\ell_{i}^{+}$makes with $\ell_{i}^{-}$, $i=1,2$. The meaning of parameter $r$ concerns to the underlying complex structure of the surface.

\section{The uniqueness results}

In this section we prove a uniqueness theorem for the surfaces $\left.X_{\theta r}, r \in\right]-1, r_{\theta}$, $\theta \in] 0, \pi[$. For the uniqueness in case $\theta=0$, see the remark on page 370 .

From a topological point of view, embeddedness is a very natural assumption that plays a fundamental role in some geometrical arguments. For this reason, we restrict our attention to the embedded case. From a technical point of view, some arguments in Subsection 3.1 (see Lemma 3.4) only work under this topological assumption.

Moreover, we also exclude case $\theta=\pi$. These surfaces are not contained in the convex hull of their boundaries, and in order to obtain the same results as in Subsection 3.1 and Subsection 3.2, this case needs to be treated slightly differently. Anyway, it can be shown that the surfaces $X_{\pi r}$ admit a similar uniqueness theorem. Furthermore, this particular family of surfaces has a very natural interpretation in the Lorentz-Minkowski three dimensional space (see [8] for details).

For the remainder of this paper, we assume that

$$
\theta \in] 0, \pi[\text {. }
$$

For $\theta \in] 0, \pi$, we consider two half-lines $r_{1}^{+}$and $r_{1}^{-}$in $\mathbb{R}^{3}$, meeting at an angle of $\theta$. Let $q_{1}^{+}$and $q_{1}^{-}$be two points in $r_{1}^{+}$and $r_{1}^{-}$, respectively, such that they are symmetric with respect the inner bisector of these half-lines. We allow $q_{1}^{+}=q_{1}^{-}$. Label by $\ell_{1}^{+}$ $\left(\ell_{1}^{-}\right)$the half-line on $r_{1}^{+}\left(r_{1}^{-}\right)$, starting at $q_{1}^{+}\left(q_{1}^{-}\right)$, and choose $q_{1}^{+}$and $q_{1}^{-}$in such a way that either $q_{1}^{+}=q_{1}^{-}$or $\ell_{1}^{+} \cap \ell_{1}^{-}=\emptyset$. 

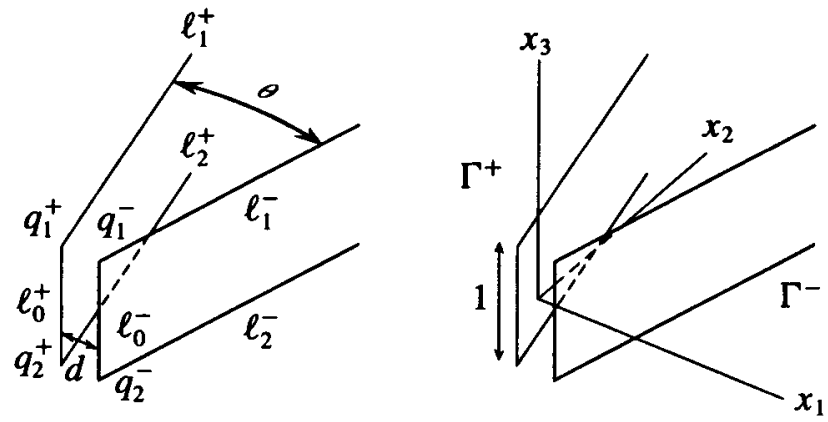

FIGURE 6. The curve $\Gamma$.

Let $\pi_{1}$ be the plane determined by $\ell_{1}^{+}$and $\ell_{1}^{-}$and label $\pi_{2}$ a plane parallel to $\pi_{1}$ and distinct. Let $\ell_{2}^{+}$and $\ell_{2}^{-}$be the orthogonal projections to $\pi_{2}$ of $\ell_{1}^{+}$and $\ell_{1}^{-}$, respectively. The orthogonal projection of $q_{1}^{+}$and $q_{1}^{-}$are denoted by $q_{2}^{+}$and $q_{2}^{-}$. We label by $\ell_{0}^{+}$ the segment $\left[q_{1}^{+}, q_{2}^{+}\right]$and $\ell_{0}^{-}$as $\left[q_{1}^{-}, q_{2}^{-}\right]$. Finally, we write

$$
\Gamma^{+}=\bigcup_{i=0}^{2}\left(\ell_{i}^{+}\right), \quad \Gamma^{-}=\bigcup_{i=0}^{2}\left(\ell_{i}^{-}\right) \text {and } \Gamma=\Gamma^{+} \cup \Gamma^{-} \text {. }
$$

Up to a homothetic transformation, we can suppose that the distance between $\pi_{1}$ and $\pi_{2}$ is 1 . We label $d$ as the distance between $\ell_{0}^{+}$and $\ell_{0}^{-}$. Notice that the possibility $\ell_{0}^{+}=\ell_{0}^{-}$is allowed, and so $d$ could be zero.

In the remainder of this paper, we use a set of Cartesian coordinates, such that

- $\ell_{0}^{+}$and $\ell_{0}^{-}$have the direction of $x_{3}$-axis;

- the planes $\pi_{1}$ and $\pi_{2}$ coincide with the planes $x_{3}=1 / 2$ and $x_{3}=-1 / 2$, respectively;

- the origin is the midpoint of $\left(q_{1}^{+}+q_{2}^{+}\right) / 2$ and $\left(q_{1}^{-}+q_{2}^{-}\right) / 2$ and;

- the $x_{2}$-axis is the inner bisector of the orthogonal projection of $\ell_{i}^{+}$and $\ell_{i}^{-}$to the plane $x_{3}=0, i=1,2$.

Throughout this section, $X: M \rightarrow \mathbb{R}^{3}$ is a proper conformal minimal immersion satisfying:

1. $M$ is homeomorphic to the closed unit disc $\overline{\mathbb{D}}$ minus two boundary points $E_{1}$ and $E_{2}$, that we call the ends of $M$. The boundary of $M$ has two connected components that we have called $\gamma^{+}$and $\gamma^{-}$.

2. $X(\partial(M))=\Gamma$.

3. If $d>0, X$ is an embedding.

4. In the limit case $\ell_{0}^{+}=\ell_{0}^{-},\left.X\right|_{M-\gamma^{+}}$and $\left.X\right|_{M-\gamma^{-}}$are injective. As a consequence, the points on $\ell_{0}=\ell_{0}^{+}=\ell_{0}^{-}$are double. (For this reason, throughout the paper, we 
will distinguish between $M$ and $X(M)$.)

5. $X(M)$ lies in the convex hull, $\mathscr{E}(\Gamma)$, of $\Gamma$.

3.1. The underlying complex structure and Weierstrass data This subsection aims at describing the complex structure of $M$ and the behaviour of the Weierstrass data. We prove that $M$ is conformally equivalent to a closed disc with piecewise analytic boundary and twice punctured at the boundary. As a consequence of this, the Gauss map and Weierstrass data will admit a meromorphic extension to the ends. The principal ideas of this subsection are inspired by Osserman's work [14].

3.1.1. The underlying complex structure. This paragraph is devoted to the study of the conformal type of the disk $M$, and the main result corresponding to Theorem 3.8. To obtain this theorem, we need some information about the Gauss map of the immersion $X$. This information is summarized in Theorem 3.1 below.

Let $\vec{a}_{2}=(0,1,0)$. Observe that, $\vec{a}_{2}$ is the unitary vector in the direction of the inner bisector of $\ell_{2}^{+}$and $\ell_{2}^{-}$pointing to $\mathscr{E}(\Gamma)$ (see Figure 6).

As a first step, we want to prove the following result.

THEOREM 3.1. There exists a neighbourhood of $\vec{a}_{2}, U\left(\vec{a}_{2}\right)$, in $\$^{2}$ such that, counting multiplicities,

$$
\sharp\left(N^{-1}(\{\vec{a},-\vec{a}\})\right)=1, \quad \forall \vec{a} \in U\left(\vec{a}_{2}\right),
$$

where $N: M \rightarrow \mathbb{S}^{2}$ is the Gauss map of $X$.

To obtain this theorem, we need additional results.

The plane $x_{2}=t$ is denoted by $\Pi_{\vec{a}_{2}}^{t}, t \in \mathbb{R}$. Notice that the fact $X(M) \subset \mathscr{E}(\Gamma)$ and the interior maximum principle imply $\Pi_{\bar{a}_{2}}^{0} \cap X(M)=\ell_{0}^{+} \cup \ell_{0}^{-}$.

DEFINITION 3.1. For $t \geq 0$, we define $\sigma_{t} \stackrel{\text { def }}{=} X^{-1}\left(X(M) \cap \Pi_{\vec{a}_{2}}^{t}\right)$.

Taking into account that $X$ is proper and $X(M) \subset \mathscr{E}(\Gamma)$, we obtain that $\sigma_{t}$ is compact, for every $t \geq 0$. Furthermore, $\sigma_{t}$ is a set of properly immersed analytic lines, because it is the nodal set of a harmonic function.

ClAIM 3.2. $\Pi_{\bar{a}_{2}}^{0}$ and $X(M)$ are transverse along $\ell_{0}^{+} \cup \ell_{0}^{-}$. In particular, the set $\sigma_{0}$ consists of two disjoint regular curves on $\partial(M)$.

To see this, it suffices to observe that $\Pi_{\bar{a}_{2}}^{0}$ and $X(M)$ are transverse along $\ell_{0}^{+} \cup \ell_{0}^{-}$. If not, using the order of contact theorem (or the maximum principle at the boundary), we obtain that $X(M)$ has points in both sides of $\Pi_{\bar{a}_{2}}^{0}$, which is absurd because $X(M) \subset$ $\mathscr{E}(\Gamma)$

On the other hand: 


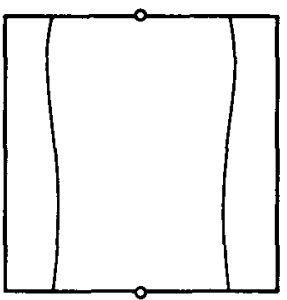

(a)

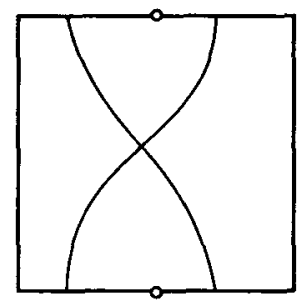

(b)

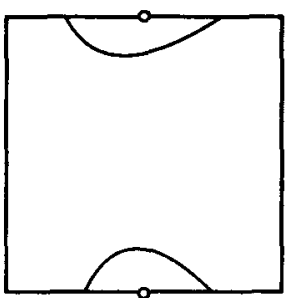

(c)

FIGURE 7. The topological possibilities for $\sigma_{t}$. (a) The ends $E_{1}, E_{2}$ are in the same connected component of $M-\sigma_{t}$. (b) $\sigma_{t}^{1}$ and $\sigma_{t}^{2}$ intersect at $P_{0} \in M$, then $X\left(P_{0}\right)$ is an ordinary point of tangential contact between $X(M)$ and $\Pi_{\hat{\sigma}_{2}}^{t}$. (c) The curves $\sigma_{t}^{1}$ and $\sigma_{t}^{2}$ are disjoint and $E_{1}, E_{2}$ are not in the same connected component of $M-\sigma_{t}$.

CLAIM 3.3. The plane $\Pi_{\vec{a}_{2}}^{\prime}$ and the surface $X(M)$ are transverse on $\ell_{1}^{+} \cup \ell_{1}^{-} \cup \ell_{2}^{+} \cup \ell_{2}^{-}$, $\forall t>0$.

Indeed, given a point in $P \in\left(\ell_{1}^{+} \cup \ell_{1}^{-} \cup \ell_{2}^{+} \cup \ell_{2}^{-}\right) \cap \Pi_{\vec{a}_{2}}^{t}$, the normal vector at $P$ lies either in the orthogonal plane to $\ell_{1}^{+}$or in the orthogonal plane to $\ell_{1}^{-}$, but $\vec{a}_{2}$ does not belong to any of both two planes, which implies that $\Pi_{\vec{a}_{2}}^{t}$ is transverse to $X(M)$ at $P$.

REMARK. Using Claim 3.3 we deduce that one, and only one, curve lying in $\sigma_{t}$, $t>0$, approaches one of the four points in $\sigma_{t} \cap \partial(M)$.

On the other hand, taking into account that $\sigma_{t}$ is compact and the interior maximum principle, we deduce there are no regions in $M$ bounded by curves in $\sigma_{t}$.

Since $M$ is simply connected, then $\sigma_{t}$ is the union of two regular simple curves, $\sigma_{t}^{1}$ and $\sigma_{t}^{2}$, with at most one point in common. Furthermore, if $\sigma_{t}^{1}$ and $\sigma_{t}^{2}$ intersect at $P_{0} \in M$, then the order of contact theorem implies that $X\left(P_{0}\right)$ is an ordinary point of tangential contact between $X(M)$ and $\Pi_{\bar{a}_{2}}^{t}$. The three topological possibilities that could occur for $\sigma_{t}$ have been illustrated in Figure 7 .

LEMMA 3.4 (Boundary behaviour). Up to relabellings, the immersion $X$ satisfies

1. $X\left(\gamma^{+}\right)=\Gamma^{+}$and $X\left(\gamma^{-}\right)=\Gamma^{-}$;

2. $X^{-1}\left(\ell_{1}^{+}\right) \cup X^{-1}\left(\ell_{1}^{-}\right)$diverges to $E_{1}$ and $X^{-1}\left(\ell_{2}^{+}\right) \cup X^{-1}\left(\ell_{2}^{-}\right)$diverges to $E_{2}$.

PROOF. If $d=\operatorname{dist}\left(\ell_{0}^{+}, \ell_{0}^{-}\right)>0$, then Statement 1 of Lemma 3.4 holds by an easy connectedness argument.

In the case $\theta>0$ and $d=0$, suppose that $X\left(\gamma^{+}\right) \neq \Gamma^{+}$. As $X\left(\gamma^{+}\right)$is a connected divergent curve over $X(\partial M)$, then $X\left(\gamma^{+}\right)$must be, up to relabellings, $\ell_{1}^{+} \cup \ell_{0} \cup \ell_{2}^{-}$, and so $X\left(\gamma^{-}\right)=\ell_{1}^{-} \cup \ell_{0} \cup \ell_{2}^{+}$. 
Label by $\gamma_{0}^{+}, \gamma_{0}^{-}$the two connected components of $X^{-1}\left(\ell_{0}\right)$. From Claim 3.2, both components are two regular arcs in $\partial(M)$ and $\Pi_{\bar{a}_{2}}^{0}$ is transverse along $\ell_{0}$. Thus, there exist two open neighbourhoods $U\left(\gamma_{0}^{+}\right), U\left(\gamma_{0}^{-}\right)$in $M$ of $\gamma_{0}^{+}, \gamma_{0}^{-}$, respectively, such that $X\left(U\left(\gamma_{0}^{+}\right)\right), X\left(U\left(\gamma_{0}^{-}\right)\right)$are graphs over $\Pi_{\bar{a}^{2}}^{0}$. In particular, there exists $t^{\prime}>0$ small enough such that, for each $t<t^{\prime}, X\left(\sigma_{t}\right)$ consists of two curves, one of them starting at $\ell_{1}^{+}$and ending at $\ell_{2}^{-}$, and the other one starting at $\ell_{1}^{-}$and ending at $\ell_{2}^{+}$. As both curves lie in $\Pi_{\hat{a}_{2}}^{t}$, then they meet at least one point. This means that $X(M)-\ell_{0}$ presents self-intersections, which is absurd.

In order to prove Assertion 2, recall that $M$ is homeomorphic to a closed disc minus two boundary points, and so, Assertion 1 in the lemma implies that $X^{-1}\left(\ell_{1}^{+}\right)$ and $X^{-1}\left(\ell_{2}^{+}\right)$diverge each other to different ends, and the same happens for $X^{-1}\left(\ell_{1}^{-}\right)$ and $X^{-1}\left(\ell_{2}^{-}\right)$.

If $\ell_{1}^{+}$and $\ell_{2}^{-}$diverge to the same end, then, without loss of generality, we can assume that $\ell_{1}^{+}, \ell_{2}^{-}$diverge to $E_{1}$, and so $\ell_{1}^{-}, \ell_{2}^{+}$diverge to $E_{2}$. Let $U\left(E_{i}\right), i=1,2$, be two open disjoint neighbourhoods of the ends. As $X$ is proper, then there is a $t^{\prime \prime}>0$ large enough such that, for every $t>t^{\prime \prime} \sigma_{t} \subset U\left(E_{1}\right) \cup U\left(E_{2}\right)$.

Therefore, the remark on page 375 says that $\sigma_{t}$ consists of two disjoint regular arcs, $\sigma_{t}^{1}$ and $\sigma_{t}^{2}$, where $\sigma_{t}^{i} \subset U\left(E_{i}\right), i=1,2$. It is clear that $X\left(\sigma_{t}^{1}\right)$ is a curve starting at $\ell_{1}^{+}$and ending at $\ell_{2}^{-}$, and $X\left(\sigma_{1}^{2}\right)$ starts at $\ell_{1}^{-}$and ends at $\ell_{2}^{+}$. As both curves lie in $\Pi_{\vec{a}_{2}}^{t} \cap \mathscr{E}(\Gamma)$, then they intersect, which is contrary to our assumptions.

In what follows, we label $\gamma_{i}^{+}=X^{-1}\left(\ell_{i}^{+}\right), \gamma_{i}^{-}=X^{-1}\left(\ell_{i}^{-}\right), i=1,2$. We define also $\gamma_{0}^{+}=X^{-1}\left(\ell_{0}^{+} \cup \ell_{0}^{-}\right) \cap \gamma^{+}$and $\gamma_{0}^{-}=X^{-1}\left(\ell_{0}^{+} \cup \ell_{0}^{-}\right) \cap \gamma^{-}$.

Observe that

$$
\gamma^{+}=\gamma_{0}^{+} \cup \gamma_{1}^{+} \cup \gamma_{2}^{+}, \quad \gamma^{-}=\gamma_{0}^{-} \cup \gamma_{1}^{-} \cup \gamma_{2}^{-} .
$$

The following lemma is a consequence of the remark on page 375 and basic facts from the Morse Theory. We include the proof for completeness.

LEMMA 3.5. Counting multiplicities, $\sharp\left[N^{-1}\left(\left\{-\vec{a}_{2}, \vec{a}_{2}\right\}\right)\right]=1$.

ProOF. Let us call $\mathscr{A}=\left\{t \in \mathbb{R}^{+} / E_{1}, E_{2}\right.$ lie in the closure of the same connected component of $\left.\left(M-\sigma_{t}\right)\right\}$, and $\mathscr{B}=\left\{t \in \mathbb{R}^{+} / \gamma_{0}^{+}, \gamma_{0}^{-}\right.$are in the same connected component of $\left.\left(M-\sigma_{t}\right)\right\}$.

Observe that $\mathscr{A}$ and $\mathscr{B}$ are not void. For, note that Claim 3.2 yields $0 \in \mathscr{A}$. Moreover, in the proof of Lemma 3.4 we have seen that for $t>0$ large enough, $\sigma_{t}$ consists of two disjoint arcs belonging to two disjoint small neighbourhoods of the ends. As we can choose these neighbourhoods not intersecting $\gamma_{0}^{+} \cup \gamma_{0}^{-}$, then $t \in \mathscr{B}$.

From the remark on page 375, it is not hard to see that $\mathscr{A} \cap \mathscr{B}=\emptyset$. Furthermore, $t \in\left[0,+\infty\left[-(\mathscr{A} \cup \mathscr{B})\right.\right.$ if and only if $\sigma_{t}$ is connected and contains a unique point of ordinary contact with normal vector in $\left\{-\vec{a}_{2}, \vec{a}_{2}\right\}$. 
The set $\left\{\sigma_{t}, t \in[0,+\infty[\}\right.$ consists of the level curves of the proper Morse function $\left\langle X, \vec{a}_{2}\right\rangle$. Since $M$ is a closed disc minus two boundary points, basic Morse theory (see Claim 3.2, Claim 3.3 and the remark on page 375) gives that $\mathscr{A}=\left[0, t_{0}[\right.$ and $\mathscr{B}=] t_{0},+\infty\left[, t_{0}>0\right.$. Moreover, $\sigma_{t_{0}}$ contains a unique point of ordinary contact and $\sigma_{t}, t \neq t_{0}$, do not. This concludes the proof.

PROOF OF THEOREM 3.1. Let $\beta:] 0,1[\rightarrow M-\partial(M)$ be a continuous curve which diverges to both ends $E_{1}$ and $E_{2}$. Define $\mathscr{A}$ and $\mathscr{B}$ as in the proof of Lemma 3.5.

Let $t$ be a point in $\mathscr{A}$, such that $\sigma_{t} \cap \beta(10,1[)=\emptyset$. Then, as we have seen during the proof of Lemma 3.5, there exists $\epsilon>0$ such that $t-\epsilon>0$ and $[0, t+\epsilon[\subset \mathscr{A}$. As $X$ is proper, we can choose $\epsilon$ small enough such that $L \cap X(\beta(] 0,1[))=\emptyset$, where $L$ is the open slab in $\mathbb{R}^{3}$ bounded by $\Pi_{\vec{a}_{2}}^{t-\epsilon}$ and $\Pi_{\vec{a}_{2}}^{t+\epsilon}$.

Let $\mathscr{P}$ be the set of planes $\Pi \subset \mathbb{R}^{3}$ such that $\Pi \cap \Gamma \subset L \cap \Gamma$, and label $V=\{\vec{a}(\Pi) / \Pi \in \mathscr{P}\} \subset \mathbb{S}^{2}$, where $\vec{a}(\Pi)$ is the unit normal vector to $\Pi$ satisfying $\left\langle\vec{a}(\Pi), \vec{a}_{2}\right\rangle \geq 0$. It is clear that $V$ is an open set in $\mathbb{S}^{2}$, containing $\vec{a}_{2}=\vec{a}\left(\Pi_{\vec{a}_{2}}^{0}\right)$. From Claim 3.2, Claim 3.3 and shrinking $L$ if necessary, we can assume that any plane in $\mathscr{P}$ is transverse to $X(M)$ along $\Gamma$.

On the other hand, let $\mathscr{H}$ be the closed halfspace bounded by $\Pi_{\bar{a}_{2}}^{t+\epsilon}$ with inner normal vector $-\vec{a}_{2}$. As $X$ is proper, then the set $\mathscr{C}=X^{-1}(\mathscr{H})$ is compact. Moreover, since $t \in \mathscr{A}$, then the proof of Lemma 3.5 gives $\pm \vec{a}_{2} \notin N(\mathscr{C})$. As $N(\mathscr{C})$ is a compact subset of $\mathbb{S}^{2}$, then there exists an open neighbourhood $V^{\prime}$ of $\vec{a}_{2}$ such that $\left( \pm V^{\prime}\right) \cap N(\mathscr{C})=\emptyset$.

Thus, $U\left(\vec{a}_{2}\right) \stackrel{\text { def }}{=} V \cap V^{\prime}$ is the neighbourhood that we are looking for. Indeed, given a unitary vector $\vec{a} \in U\left(\vec{a}_{2}\right)$, let $\Pi_{\vec{a}}^{0}$ be a plane orthogonal to $\vec{a}$ and contained in $\mathscr{P}$, and for any $t \geq 0$, define $\Pi_{\vec{a}}^{t}$ as the plane parallel to $\Pi_{\vec{a}}^{0}$ whose height with respect to $\vec{a}$ is $t$.

Then, like in the proof of Lemma 3.5, we define $\mathscr{A}(\vec{a})=\left\{t \in \mathbb{R}^{+} / E_{1}, E_{2}\right.$ lie in the closure of the same connected component of $\left.\left(M-X^{-1}\left(\Pi_{\tilde{a}}^{t}\right)\right)\right\}$, and $\mathscr{B}(\vec{a})=\{t \in$ $\mathbb{R}^{+} / \gamma_{0}^{+}, \gamma_{0}^{-}$are in the same connected component of $\left.\left(M-X^{-1}\left(\Pi_{\vec{a}}^{t}\right)\right)\right\}$. Reasoning like in that proof, it is not hard to get $\mathscr{A}(\vec{a})=\left[0, t_{0}[\right.$ and $\mathscr{B}(\vec{a})=] t_{0},+\infty[$, where $t_{0}>0$. Therefore, there exists a unique point of ordinary contact in $\Pi_{\bar{a}}^{t_{0}}$, and there are no such points in $\Pi_{\vec{a}}^{t}, t \neq t_{0}$.

Consider $\mathscr{B}$ as in the proof of Lemma 3.5.

DEFINITION 3.2. For $t \in \mathscr{B}$, we define $D_{i}^{i}$ as the closure of the connected component of $M-\sigma_{t}$ containing $E_{i}, i=1,2$, where $\sigma_{t}=X^{-1}\left(X(M) \cap \Pi_{\bar{a}_{2}}^{t}\right)$.

Observe that $D_{t}^{i}$ is homeomorphic to $\overline{\mathbb{D}}-\{1\}$, and its boundary is the union of three analytic curves 'laid end to end'. One of them is, up to relabelling, $\sigma_{t}^{i}$ (see the remark on page 375 ), and the other two, $a_{t}^{i}$ and $b_{t}^{i}$, are divergent curves lying in $\partial(M)$. 


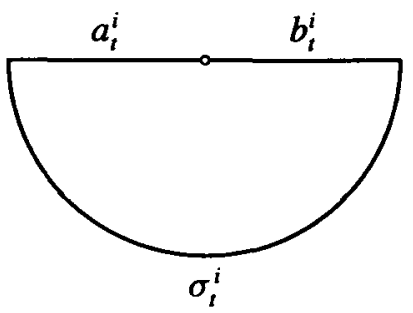

FIGURE 8. The topology of $D_{i}^{i}$.

For the remainder of this paragraph, we use a set of coordinates such that $\vec{a}_{2}=$ $(0,0,1)$.

As $\Pi_{\vec{a}_{2}}^{t}$ is transverse to $X(M)$ for every $t \in \mathscr{B}$, we deduce that:

REMARK. For any $t \in \mathscr{B}$, the map $g$ has neither zeroes nor poles on $D_{i}^{i}, i=1,2$ and so $\eta g$ has no zeroes in these sets.

Let $X_{3}: M \rightarrow \mathbb{R}$ be the third coordinate function, and let $X_{3}^{*}$ its conjugate harmonic function. Thus, $h=X_{3}+i X_{3}^{*}$ is a holomorphic function satisfying $h^{\prime}(z) d z=\eta g$, where $z$ is a conformal parameter in $D_{t}^{i}$. In particular, from the preceding remark one has

$$
h^{\prime}(z) \neq 0, \quad \text { for every } z \in D_{t}^{i}, i=1,2 \text {. }
$$

LEMMA 3.6. For any $t \in \mathscr{B}$, the map $h: D_{t}^{i} \rightarrow h\left(D_{t}^{i}\right)$ is bijective.

PROOF. Since $\left.\left(X_{3}\right)\right|_{D_{i}^{i}} ^{-1}(s)=\sigma_{s}^{i}$, it suffices to prove that $\left.\left(X_{3}^{*}\right)\right|_{\sigma_{s}^{i}}$ is injective, for every $s \geq t$.

On the other hand, as $\sigma_{s}^{i}$ is a regular curve and $h^{\prime}$ never vanishes, then $X_{3}^{*}$ is monotone on $\sigma_{s}^{i}$. This concludes the proof.

Lemma 3.6 implies that $h$ is a biholomorphism from $D_{t}^{i}-\partial\left(D_{t}^{i}\right)$ onto $h\left(D_{t}^{i}-\partial\left(D_{t}^{i}\right)\right)$, and (8) implies that it extends to the boundary in a regular way.

Now, we can prove the following:

LEMMA 3.7. For $t \in \mathscr{B}$ large enough, we have

$$
R<|g(P)|<1 / R, \quad \text { for every } P \in D_{t}^{i}, i=1,2 \text {, }
$$

where $R \in] 0,1[$ is a suitable constant depending on $t$. 
PROOF. Let $U$ be the stereographic projection of $U\left(\vec{a}_{2}\right) \cup\left(-U\left(\vec{a}_{2}\right)\right)$ over the Riemann sphere. Taking into account the choice of the frame, $U$ is a neighbourhood of $\{0, \infty\}$ in $\overline{\mathbb{C}}=\mathbb{C} \cup\{\infty\}$.

Let $P_{0}$ be the unique point of $M$ such that $g\left(P_{0}\right) \in\{0, \infty\}$ (see Theorem 3.1). As $g$ is continuous, there is a compact neighbourhood $\bar{V}$ of $P_{0}$ satisfying

$$
g(\bar{V}) \cup\left(-\frac{1}{\bar{g}}\right)(\bar{V}) \subset U
$$

If we define

$$
K \stackrel{\text { def }}{=} g(\bar{V}) \cup\left(-\frac{1}{\bar{g}}\right)(\bar{V}),
$$

then $K$ is a compact neighbourhood of $g\left(P_{0}\right)$. Using Theorem 3.1, we deduce that $g^{-1}(K)=\bar{V}$, and so $g(M-\bar{V}) \cap K=\emptyset$.

If we take $t$ large enough such that $D_{t}^{i} \cap \bar{V}=\emptyset$, then $g\left(D_{t}^{i}\right) \cap K=\emptyset, i=1,2$.

Since $K$ is a neighbourhood of $\{0, \infty\}$, we can guarantee the existence of a constant $1>R>0$ satisfying the statement of the lemma.

For the remainder of the paragraph, we fix $t \in \mathscr{B}$ as in Lemma 3.7, and for the sake of simplicity, we write $D^{i}, \sigma^{i}, a^{i}$ and $b^{i}$ instead of $D_{t}^{i}, \sigma_{t}^{i}, a_{t}^{i}$ and $b_{t}^{i}$, respectively.

If we consider Weierstrass data $(g, \eta)$ on $D^{i}$, then the induced metric

$$
d \hat{s}^{2}=\frac{1}{4}\left(1+|g|^{2}\right)^{2}|\eta|^{2}
$$

is complete.

We can now state the main result of this paragraph.

THEOREM 3.8 (Underlying complex structure). The neighbourhood $D^{i}-\sigma^{i}$ is conformally diffeomorphic to $\mathbb{D}^{+}-\{0\}$, where

$$
\mathbb{D}^{+}=\{z \in \mathbb{C} /|z|<1, \operatorname{Im}(z) \geq 0\} .
$$

In particular, the Riemann surface $M$ is conformally diffeomorphic to a closed disc with piecewise analytic boundary $\bar{M}$ minus two boundary points $\left\{E_{1}, E_{2}\right\}$.

PROOF. We split the proof in several claims.

ClAIM 3.9. The bilinear form $d s^{2} \stackrel{\text { def }}{=}|\eta g|^{2}$ is a complete metric on $D^{i}$.

As $g$ has neither zeroes nor poles in $D^{i}$, then $\eta g$ has no zeroes on $D^{i}$. Taking Lemma 3.7 into account we deduce that $d \hat{s} \leq R d s$. As $d \hat{s}^{2}$ is complete, then $d s^{2}$ is complete too. 
ClaIM 3.10. The map

$$
h:\left(D^{i}, d s^{2}\right) \rightarrow\left(h\left(D^{i}\right), d s_{0}^{2}\right)
$$

is an isometry, where $d s_{0}{ }^{2}$ represents the Euclidean metric in the complex plane. Hence, $\left(h\left(D^{i}\right), d s_{0}{ }^{2}\right)$ is complete.

To see this, let us consider $\lambda(s)$ a differentiable curve in $D^{i}$, then one has

$$
L_{d s^{2}}(\lambda)=\int_{\lambda}|\eta g|=\int_{\lambda}\left|h^{\prime}(z) d z\right|=L_{d s_{0}^{2}}(h \circ \lambda) .
$$

This means that $h$ is a length-preserving map. In view of Lemma 3.6, this implies that $h$ is an isometry.

ClAIM 3.11. $h\left(a^{i}\right)$ and $h\left(b^{i}\right)$ are divergent curves in $\mathbb{C}$.

For, it suffices to recall that $X\left(a^{i}\right)$ and $X\left(b^{i}\right)$ are divergent pieces of straight lines in $\Gamma$, and so our choice of the frame gives that $\operatorname{Re}\left(h\left(a^{i}\right)\right)=X_{3}\left(a^{i}\right)$ and $\operatorname{Re}\left(h\left(b^{i}\right)\right)=$ $X_{3}\left(b^{i}\right)$ diverge to $+\infty, i=1,2$.

From Claim 3.10 and Claim 3.11, we deduce that $h\left(D^{i}\right)$ is a simply connected region in $\overline{\mathbb{C}}$, and

$$
\partial\left(h\left(D^{i}\right)\right)=h\left(\sigma^{i}\right) \cup h\left(a^{i}\right) \cup h\left(b^{i}\right), \quad i=1,2 .
$$

Furthermore, these three curves 'laid end to end', and

- $h\left(\sigma^{i}\right)$ is compact;

- $h\left(a^{i}\right)$ and $h\left(b^{i}\right)$ diverge to $\infty$.

Hence, $D^{i}$ is conformally diffeomorphic to a closed disc with piecewise analytic boundary, and once punctured at the boundary, $i=1,2$.

Finally, let us observe that $D_{i}-\sigma_{i}$ is biholomorphic to $\mathbb{D}^{+}-\{0\}$.

Let $\widetilde{X}: \widetilde{D}^{i} \rightarrow \mathbb{R}^{3}$ be the conformal minimal immersion obtained from $X\left(D^{i}-\sigma^{i}\right)$ by successive Schwarz reflections about straight lines. Here, $\widetilde{D}^{i}$ is an open Riemann surface, that is $\partial\left(\widetilde{D}^{i}\right)=\emptyset$.

Label $J: \widetilde{D}^{i} \rightarrow \widetilde{D}^{i}$ as the automorphism induced by the rotation about the $x_{3}$-axis by angle $2 \theta$. Let $q: \widetilde{D}^{i} \rightarrow \widetilde{D}^{i} /\langle J\rangle$ be the natural projection, and observe that $\widetilde{D}^{i} /\langle J\rangle$ is conformally equivalent to $\mathbb{D}^{*}=\mathbb{D}-\{0\}$.

Represent by $\widetilde{S}$ the antiholomorphic involution on $\widetilde{D}^{i}$ induced by the 180 degrees rotation about a straight line in $\widetilde{X}\left(\widetilde{D}^{i}\right)$. Then it is easy to see that $J \circ \widetilde{S}=\widetilde{S} \circ J$. This implies that $\tilde{S}$ induces an antiholomorphic involution $S$ on the quotient $\tilde{D}^{i} /\langle J\rangle \equiv \mathbb{D}^{*}$, fixing 0 . Then, up to a conformal transformation, $S(z)=\bar{z}$, and so $D_{i}-\sigma_{i}$ is biholomorphic to $\mathbb{D}^{+}-\{0\}$. This completes the proof. 
3.1.2. The Weierstrass data. In this paragraph we prove that the Gauss map and Weierstrass data extend continuously to the ends (see Theorem 3.12 for details). As a consequence, the immersion $X$ has finite total curvature.

At this point we return to the original frame given at the beginning of this section. In particular, if we write $X(P)=\left(X_{1}(P), X_{2}(P), X_{3}(P)\right)$, one has $\left.X_{3}\right|_{\gamma_{2}^{+} \cup \gamma_{2}^{-}}=-1 / 2$ and $\left.X_{3}\right|_{y_{1}^{+} \cup y_{1}^{-}}=1 / 2$.

THEOREM 3.12. The maps $X_{3}$ and $g$ extend continuously to $E_{i}, i=1,2$. In particular, the total curvature of $M$ is finite.

Furthermore the limit tangent plane to $M$ at $E_{i}$ is $\pi_{i}, i=1,2$.

PROOF. The harmonic function $X_{3}$ is bounded, and $\left.X_{3}\right|_{\gamma_{i}^{+}} u_{\gamma_{i}^{-}}$is constant. From Theorem 3.8, $X_{3}$ extends continuously in a natural way to $E_{i}, i=1,2$.

For the second part of Theorem 3.12, we need to prove several claims.

ClAIM 3.13. Let $Q_{i} \in \gamma_{i}^{+}$(respectively $\gamma_{i}^{-}$), and let $\Sigma_{i}$ be the tangent plane to $X(M)$ at $X\left(Q_{i}\right), i=1,2$.

Denote by $\mathscr{H}_{i}$ the closed halfspace bounded by $\Sigma_{i}$ which contains planes parallel to $\Sigma_{i}$ and disjoint to $\mathscr{E}(\Gamma)$ (and so, disjoint to $X(M)$ ).

Then, there are no domains $\Omega_{i} \subset M-\partial(M)$ such that

- $\partial\left(\Omega_{i}\right) \subset X^{-1}\left(\Sigma_{i} \cap X(M)\right)$;

- $X\left(\Omega_{i}\right)$ lies on the closed halfspace $\mathscr{H}_{i}$.

Indeed, if a such domain exists, then the distance from points of $\Omega_{i}$ to $\Sigma_{i}$ is bounded. So, the claim is an easy consequence of [11, Lemma 2.1].

ClAIM 3.14. For any $P \in \gamma_{1}^{+} \cup \gamma_{1}^{-} \cup \gamma_{2}^{+} \cup \gamma_{2}^{-}$, counting multiplicities, one has

$$
\sharp\left[g^{-1}(g(P))\right]<9 .
$$

We prove this claim in the case $X(P) \in \ell_{1}^{+}$. The proofs of the other three possibilities are similar.

Label by $\Sigma$ the tangent plane to $X(M)$ at $X(P)$, and observe that $\ell_{1}^{+} \subset \Sigma$. Let us consider $\Delta=X^{-1}(\Sigma \cap X(M))$. Since $\Delta$ is the nodal set of a harmonic function, then $\Delta$ is a set of properly immersed analytic curves.

It is straightforward to prove that $\Delta \cap X^{-1}\left(\Gamma-\ell_{1}^{+}\right)$has at most two points, one of them in $\gamma_{0}^{-}$and the other one in $\gamma_{2}^{-}$. In particular, $\partial(M)-\Delta$ has at most four connected components.

On the other hand, the interior maximum principle gives us that there are no compact simply connected region of $M$ bounded by curves in $\Delta$. Since $M$ is simply connected, this implies that: 
- If $\delta$ is a curve in $\Delta$ starting at $\gamma_{1}^{+}$, then either $\delta$ diverges to an end or the end point of $\delta$ belongs to $X^{-1}\left(\Gamma-\ell_{1}^{+}\right) \cap \Delta$.

- When two curves in $\Delta$ originates from $\gamma_{1}^{+}$, they do not intersect.

As $M$ is simply connected, elementary topological arguments imply that $M-\Delta$ has at least as many connected components as curves in $\Delta$ approaching $\gamma_{1}^{+}$plus one. If $\sharp\left[g^{-1}(g(P))\right] \geq 9$ then, using the order of contact theorem, there are at least 9 curves in $\Delta$ starting at $P \in \gamma_{1}^{+}$. Hence, we deduce that $M-\Delta$ has at least ten connected components.

Taking into account that $\partial(M)-\Delta$ has at most four connected components, there are six components whose boundary is included in $\Delta$.

Moreover, the order of contact theorem implies that at least five components of $M-\Delta$ lie above $\Sigma$, and another five below.

Therefore, we deduce the existence of a domain $\Omega \in M-\partial(M)$ verifying

- $\partial(\Omega) \subset \Delta$;

- $X(\Omega)$ lies in the closed halfspace $\mathscr{H}$ with boundary $\Sigma$, which contains planes parallel to $\Sigma$ and disjoint to $\mathscr{E}(\Gamma)$,

which is contrary to Claim 3.13. This proves Claim 3.14.

At this point we can now prove the following:

ClaIM 3.15. The following limits exist:

$$
\lim _{\substack{P \rightarrow E_{i} \\ P \in \gamma_{+}}} g(P), \quad \lim _{\substack{P \rightarrow E_{i} \\ P \in \gamma_{-}}} g(P), \quad i=1,2 .
$$

Consider $\ell \in\left\{\ell_{1}^{+}, \ell_{1}^{-}, \ell_{2}^{+}, \ell_{2}^{-}\right]$, and let $\alpha:[0,+\infty[\rightarrow M$ be the parametrization by arc length of $X^{-1}(\ell)$.

We are trying to prove that $\lim _{s \rightarrow+\infty} f(s)$ exists, where $f=g \circ \alpha$.

First, observe that the normal vectors along $\ell$ lie in a meridian of $\mathbb{S}^{2}$. Then, $f([0,+\infty[) \subset \mathbf{w}$, where $\mathbf{w}$ is a suitable straight line in $\overline{\mathbb{C}}$. Suppose this limit does not exist. Then there are two sequences $\left\{p_{n}\right\}$ and $\left\{q_{n}\right\}$ in $[0,+\infty[$ such that:

- $\lim _{n} p_{n}=\lim _{n} q_{n}=+\infty$,

- $p_{n}<q_{n}$, for every $n \in \mathbb{N}$,

- $\lim _{n} f\left(p_{n}\right)=a, \lim _{n} f\left(q_{n}\right)=b, a, b \in \mathbf{w}, a \neq b$.

Let $c$ be a point in $w$ between $a$ and $b . \operatorname{As}_{n} f\left(p_{n}\right)=a$ and $\lim _{n} f\left(q_{n}\right)=b$, there exists $n_{0} \in \mathbb{N}$ such that

$$
\left\{c,-\frac{1}{\bar{c}}\right\} \cap f(] p_{n}, q_{n}[) \neq \emptyset, \quad \text { for } n \geq n_{0},
$$

which is contrary to Claim 3.14 .

Taking into account Theorem 3.8 and the Uniformization Theorem, we deduce that $M$ is conformally equivalent to a closed disk minus two boundary points (or a sector 
$\left.\left\{z \in \mathbb{C}^{*}: \arg (z) \in\left[t_{1}, t_{2}\right]\right\}\right)$, where the biholomorphism extends piecewise analytically to the boundary. At this point, we need the following technical result by Lindelöf:

Let $t_{1}<t_{2}, t_{2}-t_{1}<2 \pi, S=\left\{r e^{i t}: r>0, t_{1}<t<t_{2}\right\}$ and $f: \bar{S} \rightarrow \mathbb{C}$ be bounded and continuous, holomorphic in $S$. Suppose that for some $a, b \in \overline{\mathbb{C}}$ we have

$$
\lim _{r \rightarrow+\infty} f\left(r e^{i t_{1}}\right)=a, \quad \lim _{r \rightarrow+\infty} f\left(r e^{i t_{2}}\right)=b .
$$

Then $a=b$ and $\lim _{z \rightarrow \infty} f(z)=a$.

We refer the reader to [2, pages 132-133].

This theorem and Claim 3.15 imply that $g$ extends continuously to the ends.

Finally, note that $\theta>0$ implies that $g\left(\gamma_{i}^{+}\right)$and $g\left(\gamma_{i}^{-}\right)$lie in different great circles of $\$^{2}$. Thus, $g\left(\gamma_{i}^{+}\right) \cap g\left(\gamma_{i}^{-}\right) \subset\{0, \infty\}$ and so the limit normal vectors at the ends must be vertical, that is the limit tangent plane at $E_{i}$ coincides with $\pi_{i}, i=1,2$. This concludes the proof.

As a consequence of Theorem 3.12, one has the following corollary.

COROLlARY 3.16. For $t \in \mathscr{B}$ large enough $X\left(D_{t}^{i}\right)$ is a graph over the plane $\pi_{i}$, $i=1,2$.

PROOF. Take $t \in \mathscr{B}$ large enough and such that $g\left(D_{t}^{i}\right)$ does not intersect the equator $\{z \in \overline{\mathbb{C}} /|z|=1\}$.

Label $p_{3}$ as the orthogonal projection from $X(M)$ to the plane $x_{3}=0$. Taking into account that $X(M) \subset \mathscr{E}(\Gamma)$ and the definition of $D_{i}^{i}$, it is not hard to see that $\left.p_{3}\right|_{X\left(D_{i}^{i}\right)}$ is a local diffeomorphism onto $\Omega_{t}^{i}$, where $\Omega_{i}^{i}$ is the unbounded convex domain in the plane $x_{3}=0$ limited by $\mathfrak{p}_{3}\left(\ell_{i}^{+}\right), \mathfrak{p}_{3}\left(\ell_{i}^{-}\right)$and $\mathfrak{p}_{3}\left(\sigma_{i}^{i}\right)=\left\{\left(x_{1}, x_{2}, x_{3}\right) \in \mathbb{R}^{3} / x_{3}=0, x_{2}=t\right\}$.

As $X$ is proper, then the same occur for the map $\left.\mathfrak{p}_{3}\right|_{X\left(D_{l}^{i}\right)}$. So, $\left.\mathfrak{p}_{3}\right|_{X\left(D_{t}^{i}\right)}$ is a covering map, and taking into account that $\Omega_{t}^{i}$ is simply connected we deduce that $\left.\mathfrak{p}_{3}\right|_{X\left(D_{i}^{i}\right)}$ is one-to-one. This concludes the proof.

3.2. The symmetries of the surface. The aim of this subsection is to show that $X(M)$ possesses the same planes of symmetry that $\Gamma$. So, we generalize the ideas used by Schoen in [16] to our particular case of non-compact piecewise analytic boundary.

3.2.1. The vertical symmetry. In this paragraph with the frame fixed at the beginning of Section 3, we prove that $X(M)$ is invariant under symmetry with respect to the plane $x_{1}=0$.

We need to introduce some notation. For $t \geq 0$ we define

1. $\delta_{t}=X(M) \cap\left\{x_{1}=t\right\}$,

2. $M_{+}(t)=\left\{\left(x_{1}, x_{2}, x_{3}\right) \in X(M) / x_{1} \geq t\right\}$,

3. $M_{-}(t)=\left\{\left(x_{1}, x_{2}, x_{3}\right) \in X(M) / x_{1} \leq t\right\}$, 
4. $M_{+}^{*}(t)=\left\{\left(2 t-x_{1}, x_{2}, x_{3}\right) /\left(x_{1}, x_{2}, x_{3}\right) \in M_{+}(t)\right\}$, symmetric to $M_{+}(t)$ with respect to the plane $\left\{x_{1}=t\right\}$,

5. $M_{-}^{*}(t)=\left\{\left(2 t-x_{1}, x_{2}, x_{3}\right) /\left(x_{1}, x_{2}, x_{3}\right) \in M_{-}(t)\right\}$, symmetric to $M_{-}(t)$ with respect to the plane $\left\{x_{1}=t\right\}$.

In general, given a set $A \subset \mathbb{B}^{3}$, we denote by $A_{+}(t)\left(A_{-}(t)\right)$ the elements of $A$ on and above (on and below) $\left\{x_{1}=t\right\}$, and $A_{+}^{*}(t)\left(A_{-}^{*}(t)\right)$ its reflection about the plane $\left\{x_{1}=t\right\}$.

Let $\mathfrak{p}_{1}: \mathbb{R}^{3} \rightarrow\left\{x_{1}=0\right\}$ denote the orthogonal projection.

If $A, B \subset \mathbb{R}^{3}$, we say that $A \geq B$ provided for every $x \in\left\{x_{1}=0\right\}$ for which $\mathfrak{p}_{1}^{-1}(\{x\}) \cap A \neq \emptyset$ and $\mathfrak{p}_{1}^{-1}(\{x\}) \cap B \neq \emptyset$, we have

$$
\text { Infimum }\left[x_{1}\left(\mathfrak{p}_{1}^{-1}(\{x\}) \cap A\right)\right] \geq \operatorname{Supremum}\left[x_{1}\left(\mathfrak{p}_{1}^{-1}(\{x\}) \cap B\right)\right] .
$$

THEOREM 3.17. The surface $X(M)$ is symmetric with respect to the plane $\left\{x_{1}=0\right\}$, and $M_{+}(0)$ and $M_{-}(0)$ are graphs over $\left\{x_{1}=0\right\}$.

Proof. For any $t>0$, define

$$
\Delta_{t}=M_{+}^{*}(t) \cap M_{-}(t) .
$$

CLAIM 3.18. For any $t>0, \Delta_{t} \cap\left(\Gamma_{+}^{*}(t) \cup \Gamma_{-}(t)\right)=\delta_{t} \cap \Gamma$.

Since $X(M) \subset \mathscr{E}(\Gamma)$ and $t>0$, then $\Gamma_{-}(t) \cap M_{+}^{*}(t)=\delta_{t} \cap \Gamma$.

Furthermore, by the interior maximum principle, and using once again that $X(M) \subset$ $\mathscr{E}(\Gamma)$, no points of $X(M)-\Gamma$ lie on the boundary of $\mathscr{E}(\Gamma)$. So, it is not hard to deduce that $\Gamma_{+}^{*}(t) \cap M_{-}(t)=\delta_{t} \cap \Gamma$.

Taking these facts into account, we deduce easily that $\Delta_{t}-\delta_{t}$ cannot intersect $\Gamma_{-}(t) \cup \Gamma_{+}^{*}(t)$, which proves the claim.

Define now

$$
\mathscr{I}_{1}=\{t \in] 0,+\infty\left[/ M_{+}(t) \text { is a graph over the }\left(x_{2}, x_{3}\right) \text {-plane and } M_{+}^{*}(t) \geq M_{-}(t)\right\} \text {. }
$$

We want to prove that $\left.\mathscr{I}_{1}=\right] 0,+\infty[$. To do this, we need several results.

Let $s_{0}>0, s_{0} \in \mathscr{B}$, and consider (Definition 3.2) $D_{s_{0}}^{i}$ as the closure of the connected component of $M-\sigma_{s_{0}}$ containing $E_{i}, i=1,2$, where $\sigma_{s_{0}}=X^{-1}\left(X(M) \cap\left\{x_{2}=s_{0}\right\}\right)$.

If $s_{0}>0$ is large enough, then, by Theorem 3.12 and Corollary 3.16, $X\left(D_{s_{0}}^{i}\right)$ is a graph over the $\left(x_{1}, x_{2}\right)$-plane, $i=1,2$, and

$$
\left.x_{3}\right|_{x\left(D_{s_{0}}^{1}\right)}>0,\left.\quad x_{3}\right|_{x\left(D_{s_{0}}^{2}\right)}<0 .
$$

See Corollary 3.16 for details. For simplicity label $D^{i}=D_{s_{0}}^{i}-\sigma_{s_{0}}^{i}, i=1,2$.

ClaIM 3.19. If $X^{-1}\left(\Delta_{t}-\delta_{t}\right) \subset D^{1} \cup D^{2}$, then $\Delta_{t}=\delta_{t}$. 
Suppose that $\Delta_{t}-\delta_{t} \neq \emptyset$. Since $\Delta_{t}$ is locally the intersection of distinct minimal surfaces, it follows that $\Delta_{t}$ is a one-dimensional real analytic variety, properly immersed in $\mathbb{R}^{3}$.

On the other hand, as $X\left(D^{i}\right)$ are graphs over the plane $x_{3}=0$ and the projection of $X\left(D^{i}\right)$ onto this plane is a convex domain, then $X^{-1}\left(\delta_{t}\right) \cap D^{i}$ consists of a simple arc diverging to $E_{i}, i=1,2$. Taking into account Claim 3.18, we have

$$
\left.X^{-1}\left(\Delta_{t}-\delta_{t}\right)\right) \subset\left(\left(D^{1} \cup D^{2}\right)-\partial(M)\right) .
$$

Moreover, since any curve in $\Delta_{t}-\delta_{t}$ is properly immersed in $\mathbb{R}^{3}$, then no such curve diverges to an interior point in $\sigma_{s_{0}}^{i}, i=1,2$.

On the other hand, the definition of $D^{i}$ and (9) yield that

$$
X^{-1}\left(\Delta_{t}\right) \cap D^{i}=X^{-1}\left(X\left(D^{i}\right)_{+}^{*}(t) \cap X\left(D^{i}\right)_{-}\right) .
$$

So, $X^{-1}\left(\Delta_{t}-\delta_{t}\right) \cap D^{i} \neq \emptyset$ implies

$$
X^{-1}\left(\Delta_{t}-\delta_{t}\right) \cap D^{i}=X^{-1}\left(X\left(D^{i}\right)_{+}^{*}(t) \cap X\left(D^{i}\right)_{-}\right)-\partial(M) \neq \emptyset .
$$

Therefore, it is not hard to see that there exist two simply connected closed regions $\Omega_{1} \subset X\left(D^{i}\right)_{-}(t)$ and $\Omega_{2} \subset X\left(D^{i}\right)_{+}^{*}(t)$ bounded by the same curve in $\Delta_{t}$ (eventually $\Omega_{i}$ could be unbounded, $i=1,2$ ).

Summarizing, $\Omega_{1}$ and $\Omega_{2}$ are bounded graphs over its common orthogonal projection $\Omega_{0}$ onto the $\left(x_{1}, x_{2}\right)$-plane, and $\partial\left(\Omega_{1}\right)=\partial\left(\Omega_{2}\right)$.

If $\Omega_{0}$ is compact, then the interior maximum principle gives a contradiction.

If $\Omega_{0}$ is unbounded, then observe that the functions on $\Omega_{0}$ which determine the graphs $\Omega_{1}$ and $\Omega_{2}$ are asymptotic to the same finite value at infinity. The maximum principle at infinity for minimal graphs leads to a contradiction once again. This proves the claim.

ClaIM 3.20. There exists $t^{\prime}>0$ large enough such that $\left[t^{\prime},+\infty\left[\subset \mathscr{I}_{1}\right.\right.$.

Let $t^{\prime}>0$ such that $X^{-1}\left(M_{+}(t)\right) \subset D^{1} \cup D^{2}$, and observe that $X^{-1}\left(\Delta_{t}\right) \subset D^{1} \cup D^{2}$, for $t \geq t^{\prime}$. Hence from Claim 3.19, $\Delta_{t}=\delta_{t}, t \geq t^{\prime}$.

It is clear that $X^{-1}\left(M_{+}(t)\right)$ consists of two simply connected components, one of them in $D^{1}$ and the other one in $D^{2}$, for $t \geq t^{\prime}$, and thus $M_{+}(t)$ is the union of two disjoint graphs $G_{+}^{1}(t)$ and $G_{+}^{2}(t)$ over the same simply connected domain $G_{+}(t)$ in the $\left(x_{1}, x_{2}\right)$-plane.

From (9), we have that $\mathfrak{p}_{1}\left(G_{+}^{1}\left(t^{\prime}\right)\right) \cap \mathfrak{p}_{1}\left(G_{+}^{2}\left(t^{\prime}\right)\right)=\emptyset$.

Let us see that $M_{+}(t)$ is a graph over the plane $\left\{x_{1}=t\right\}, t \geq t^{\prime}$.

First, observe that $\left.p_{1}\right|_{\delta_{t}}$ is injective, $t \geq t^{\prime}$. Indeed, note that $\delta_{t} \subset\left\{x_{1}=t\right\}$ is a graph over a connected piece of the straight line $x_{1}-t=x_{3}=0$, and so the function

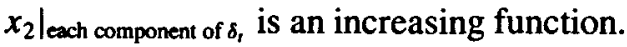




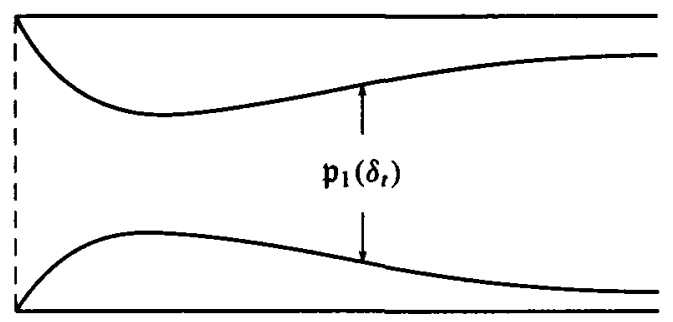

FIGURE 9. The set $\mathfrak{p}_{1}\left(M_{+}(t)\right)$.

Moreover, a similar argument gives that the set $\sigma_{s}^{i} \cap M_{+}\left(t^{\prime}\right)$ is a connected curve, for any $s>s_{0}$ (recall that, from Definition 3.2, $D^{i}=D_{s_{0}}^{i}-\sigma_{s_{0}}^{i}$ and $D_{s_{0}}^{i}$ is the closure of the connected component of $M-\sigma_{s_{0}}$ containing to $E_{i}, i=1,2$.)

On the other hand, the functions $\left.x_{3}\right|_{\sigma_{s} \cap M_{+}\left(t^{\prime}\right)}$ are monotone, for $s>s_{0}$. Otherwise, there would exist some points in $M_{+}\left(t^{\prime}\right)$ whose normal vector lie in $\left\{x_{1}=0\right\} \cap \mathbb{S}^{2}$. Thus, we could take $t \geq t^{\prime}$ in such a way that $\delta$, contains a point with normal vector in $\left\{x_{1}=0\right\} \cap \$^{2}$. Hence, from the order of contact theorem and taking into account that $X\left(D^{i}\right)$ are graphs over the plane $x_{3}=0, i=1,2$, we deduce that $\Delta_{t}-\delta_{t} \neq \emptyset$, which is absurd.

This proves that $M_{+}\left(t^{\prime}\right)$ is a graph over the plane $\left\{x_{1}=t^{\prime}\right\}$, and so the same holds for $M_{+}(t), t \geq t^{\prime}$.

Taking into account that $\Delta_{t}=\delta_{t}, t \geq t^{\prime}$ and $X(M) \subset \mathscr{E}(\Gamma)$, we deduce that $M_{+}^{*}(t) \geq M_{-}(t), t \geq t^{\prime}$. The claim holds.

It is obvious that $\mathscr{I}_{1}$ is closed in $[0,+\infty[$.

Next step demonstrates that:

Claim 3.21. The minimum of $\mathscr{I}_{1}$ is 0 .

First note that $X(M) \subset \mathscr{E}(\Gamma)$ implies that $\{0, \infty, i,-i\} \cap g(\partial(M))=\emptyset$, and so

$$
N(\partial(M)) \cap\left\{x_{1}=0\right\}=\emptyset .
$$

Assume that $t_{0}=\operatorname{Minimum}\left(\mathscr{I}_{1}\right)>0$.

Let $\mathscr{K}$ be the compact set $X\left(M-\left(D^{1} \cup D^{2}\right)\right)$. Observe that $\mathscr{K}=X^{-1}\left(\mathscr{H}_{s_{0}}\right)$, where $\mathscr{H}_{s_{0}}$ is the closed half space $x_{2} \leq s_{0}$. In particular, $M_{+}^{*}(t) \cap \mathscr{K}_{-}(t)=\mathscr{K}_{+}^{*}(t) \cap M_{-}(t)=$ $\Delta_{t} \cap \mathscr{K}$, for $t>0$.

Taking into account that $t_{0} \in \mathscr{I}_{1}$, we deduce that $\mathscr{K}_{+}\left(t_{0}\right)$ is a graph over the $\left(x_{2}, x_{3}\right)$-plane.

In particular, no point in $\mathscr{K}_{+}\left(t_{0}\right)-\left(\delta_{t_{0}} \cup \Gamma\right)$ has normal vector included in $\left\{x_{1}=0\right\}$. From equation (10) we obtain also that $N\left(\mathscr{K}_{+}\left(t_{0}\right) \cap \Gamma\right) \cap\left\{x_{1}=0\right\}=\emptyset$. Furthermore, 
the maximum principle at the boundary yields that no point in $\delta_{t_{0}} \cap \mathscr{X}_{+}\left(t_{0}\right)$ has normal vector lying in $\left\{x_{1}=0\right\}$. So, we conclude that

$$
N\left(\mathscr{X}_{+}\left(t_{0}\right)\right) \cap\left\{x_{1}=0\right\}=\emptyset .
$$

Since $\mathscr{K}_{+}\left(t_{0}\right)$ is compact, there exists $\epsilon_{0}>0$ small enough such that $t_{0}-\epsilon_{0}>0$, $N\left(\mathscr{X}_{+}(t)\right) \cap\left\{x_{1}=0\right\}=\emptyset$ and $\mathscr{K}_{+}(t)$ is a graph over the $\left(x_{2}, x_{3}\right)$-plane , for $t \in$ $\left[t_{0}-\epsilon_{0}, t_{0}\right]$.

Otherwise, we could find sequences $\left\{t_{n}\right\}$ in $\mathbb{R}^{+}$, and $\left\{P_{n}\right\},\left\{Q_{n}\right\}$ in $\mathscr{K}$, satisfying:

- $\left\{t_{n}\right\} \nearrow t_{0}$

- $P_{n}, Q_{n} \in \mathscr{K}_{+}\left(t_{n}\right), P_{n} \neq Q_{n}$ and $\mathfrak{p}_{1}\left(P_{n}\right)=\mathfrak{p}_{1}\left(Q_{n}\right)$, for every $n \in \mathbb{N}$.

- $\left\{P_{n}\right\} \rightarrow P_{0} \in \mathscr{K}_{+}\left(t_{0}\right),\left\{Q_{n}\right\} \rightarrow Q_{0} \in \mathscr{K}_{+}\left(t_{0}\right)$.

Since $\mathscr{K}_{+}\left(t_{0}\right)$ is a graph over $\left\{x_{1}=0\right\}$, the cases $P_{0} \neq Q_{0}$ and $\left\{P_{0}, Q_{0}\right\} \cap\left(\mathscr{K}_{+}\left(t_{0}\right)-\right.$ $\left.\delta_{t_{0}}\right) \neq \emptyset$ are impossible. Therefore, we would deduce that $P_{0}=Q_{0} \in \delta_{t_{0}}$, and thus, $N\left(X^{-1}\left(P_{0}\right)\right) \in\left\{x_{1}=0\right\}$. This is absurd.

In particular, $\Delta_{t} \cap \mathscr{K} \subset \mathscr{K}_{-}\left(t_{0}-\epsilon_{0}\right)$, for $t \geq t_{0}-\epsilon_{0}$.

On the other hand, $\Delta_{t_{0}}=\delta_{t_{0}}$. If not, Claim 3.18 implies that any point lying in $\Delta_{t_{0}}-\delta_{t_{0}}$ is an interior point of contact between $M_{+}^{*}\left(t_{0}\right)$ and $M_{-}\left(t_{0}\right)$. Using the interior maximum principle, we obtain $M_{+}^{*}\left(t_{0}\right)=M_{-}\left(t_{0}\right)$. This is absurd because $t_{0}>0$ and thus $\Gamma$ is not symmetric with respect to $\left\{x_{1}=t_{0}\right\}$.

Therefore, by using that $\Delta_{t_{0}}=\delta_{t_{0}}$ and that $\mathscr{K}$ is compact, we deduce the existence of $\left.\left.\epsilon_{1} \in\right] 0, \epsilon_{0}\right]$ such that $\Delta_{t} \cap \mathscr{K}=\delta_{t} \cap \mathscr{K}, t \geq t_{0}-\epsilon_{1}$.

Thus, $\Delta_{t}-\delta_{t} \subset X\left(D^{1} \cup D^{2}\right)$, and from Claim 3.19 we get $\Delta_{t}=\delta_{t}$, for $t \geq t_{0}-\epsilon_{1}$.

This implies that $M_{+}(t)$ is a graph over $\left\{x_{1}=0\right\}, \forall t \geq t_{0}-\epsilon_{1}$. Otherwise, $M_{+}\left(t_{0}-\epsilon_{1}\right)-\mathscr{K}$ would not be a graph over $\left\{x_{1}=0\right\}$. Thus, we could find two different points in $M_{+}\left(t_{0}-\epsilon_{1}\right)-\mathscr{K}$ with the same orthogonal projection on $\left\{x_{1}=0\right\}$. From the choice of $s_{0}$, both points would lie in the same $X\left(D^{i}\right), i \in\{1,2\}$. Hence, there would exists $t \geq t_{0}-\epsilon_{1}$ such that $\delta_{t} \cap X\left(D^{1} \cup D^{2}\right)$ contains points whose normal vector lie in $\left\{x_{1}=0\right\}$. So, by the order of contact theorem and using that $X\left(D^{1}\right)$ and $X\left(D^{2}\right)$ are graphs over the plane $x_{3}=0$, we would deduce that $\Delta_{t}-\delta_{t} \neq \emptyset$, which is obviously absurd.

As $X(M) \subset \mathscr{E}(\Gamma)$, then a continuity argument gives $M_{+}^{*}(t) \geq M_{-}(t), t \geq t_{0}-\epsilon_{1}$, we deduce that $t_{0}-\epsilon_{1} \in \mathscr{I}_{1}$, which contradicts that $t_{0}$ is a minimum. This proves the claim.

The last claim yields that $M_{+}^{*}(0) \geq M_{-}(0)$. A symmetric argument gives $M_{-}^{*}(0) \geq$ $M_{+}(0)$, and so the theorem holds.

3.2.2. The horizontal symmetry. Once again, we need to introduce some notation. For $t \geq-1 / 2$ we now define

(1) $\delta_{t}=X(M) \cap\left\{x_{3}=t\right\}$, 
(2) $M_{+}(t)=\left\{\left(x_{1}, x_{2}, x_{3}\right) \in X(M) / x_{3} \geq t\right\}$,

(3) $M_{-}(t)=\left\{\left(x_{1}, x_{2}, x_{3}\right) \in X(M) / x_{3} \leq t\right\}$,

(4) $M_{+}^{*}(t)=\left\{\left(x_{1}, x_{2}, 2 t-x_{3}\right) /\left(x_{1}, x_{2}, x_{3}\right) \in M_{+}(t)\right\}$,

(5) $M_{-}^{*}(t)=\left\{\left(x_{1}, x_{2}, 2 t-x_{3}\right) /\left(x_{1}, x_{2}, x_{3}\right) \in M_{-}(t)\right\}$.

Following the same notation as in Paragraph 3.2.1, given a set $A \subset \mathbb{R}^{3}$, we denote by $A_{+}(t)\left(A_{-}(t)\right)$ the elements of $A$ on and above (below) $\left\{x_{3}=t\right\}$, and $A_{+}^{*}(t)\left(A_{-}^{*}(t)\right)$ its reflection about the plane $\left\{x_{3}=t\right\}$.

Let $\mathfrak{p}_{3}: \mathbb{R}^{3} \rightarrow\left\{x_{3}=0\right\}$ denote the orthogonal projection.

If $A, B \subset \mathbb{R}^{3}$, we say that $A \geq B$ provided for every $x \in \mathbb{R}^{3}$ for which $\mathfrak{p}_{3}^{-1}(\{x\}) \cap$ $A \neq \emptyset$ and $\mathfrak{p}_{3}^{-1}(\{x\}) \cap B \neq \emptyset$, we have

$$
\text { Infimum }\left[x_{3}\left(\mathfrak{p}_{3}^{-1}(\{x\}) \cap A\right)\right] \geq \operatorname{Supremum}\left[x_{3}\left(\mathfrak{p}_{3}^{-1}(\{x\}) \cap B\right)\right] .
$$

For the sake of simplicity and in the particular case $A=M_{+}^{*}(t)-\left(\ell_{0}^{+} \cup \ell_{0}^{-}\right)$and $B=$ $M_{-}(t)-\left(\ell_{0}^{+} \cup \ell_{0}^{-}\right)$(respectively $A=M_{+}(t)-\left(\ell_{0}^{+} \cup \ell_{0}^{-}\right)$and $B=M_{-}^{*}(t)-\left(\ell_{0}^{+} \cup \ell_{0}^{-}\right)$), we write that $M_{+}^{*}(t) \succeq M_{-}(t)$ (respectively $\left.M_{+}(t) \succeq M_{-}^{*}(t)\right)$ instead of $A \geq B$, $t \in[0,1 / 2]$ (respectively $t \in[-1 / 2,0]$ ).

LEMMA 3.22. The set $\delta_{t}$ is compact, and consists of a simple arc, for every $t \in$ ] $-1 / 2,1 / 2[$. In particular, the Gauss map $g$ of $X$ omits the points 0 and $\infty$.

PROOF. From Theorem 3.12, we infer that $\delta_{t}$ is compact. Moreover, $\delta_{t} \cap \Gamma$ has two points, one of them in $\ell_{0}^{+}$and the other one in $\ell_{0}^{-}$. In case $\ell_{0}^{+}=\ell_{0}^{-}$, the only point in $\delta_{t} \cap \Gamma$ is, in a natural way, double.

Note that $X^{-1}\left(\delta_{t}\right)$ is the nodal set of the harmonic function $x_{3}-t$. Then, $X^{-1}\left(\delta_{t}\right)$ is an one-dimensional proper real analytic sub variety of $M$.

Using that $\left\{x_{3}=t\right\}$ and $X(M)$ are transverse along $\ell_{0}^{+} \cup \ell_{0}^{-}$we deduce that one, and only one, curve lying in $X^{-1}\left(\delta_{t}\right),-1 / 2<t<1 / 2$, approaches each one of the two points in $\delta_{t} \cap \partial(M)$.

On the other hand, taking into account that $X^{-1}\left(\delta_{t}\right)$ is compact and the interior maximum principle, we deduce there are no regions in $M$ bounded by curves in $X^{-1}\left(\delta_{t}\right)$.

Therefore, elementary topological arguments give that $X^{-1}\left(\delta_{t}\right)$ is a regular simple curve in $M$.

Finally, the order of contact theorem gives that there are no points in $M$ with vertical normal vector.

Define $\mathscr{E}(\Gamma)_{0}=\left\{x_{3}=0\right\} \cap \mathscr{E}(\Gamma)$, and observe that $\mathfrak{p}_{3}(X(M)) \subset \mathscr{E}(\Gamma)_{0}$

LEMMA 3.23. The map $\left.\mathfrak{p}_{3}\right|_{X(M)}: X(M) \rightarrow \mathscr{E}(\Gamma)_{0}$ is not surjective. 


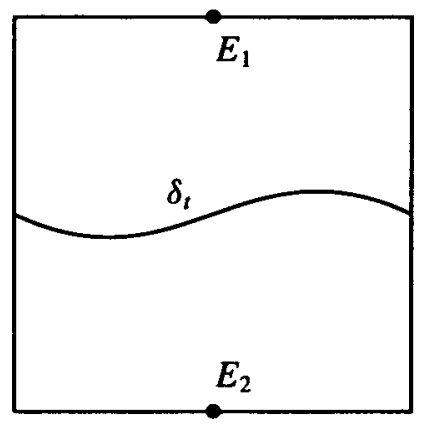

FIGURE 10. The curve $\delta_{t}$.

Moreover, there exists $\epsilon>0$ such that

$$
\mathfrak{p}_{3}\left(X^{-1}\left(\left\{x_{1}=0\right\} \cap(X(M)-\Gamma)\right)\right)=\{(0, t, 0) / t \in[\epsilon,+\infty[\} .
$$

PROOF. Consider the plane $\left\{x_{1}=0\right\}$ and denote $\alpha_{0}=X^{-1}\left(\left\{x_{1}=0\right\} \cap(X(M)-\Gamma)\right)$. Let us see that $\alpha_{0}$ consists of a single regular analytic curve diverging to the two ends of $M$.

We know that $\alpha_{0}$ is a proper one dimensional real analytic subvariety of $M-\partial(M)$. Furthermore, from the order of contact theorem, the singularities of $\alpha_{0}$ occur at the points with normal vector orthogonal to $\left\{x_{1}=0\right\}$, where a finite set of curves of $\alpha_{0}$ meet at equal angles.

From Corollary 3.16, only one curve of $\alpha_{0}$ approaches the end $E_{i}, i=1,2$.

Note also that $\alpha_{0}$ does not contain any curves approaching a point in $\partial(M)$. In case $d>0$, that is, $\ell_{0}^{+} \neq \ell_{0}^{-}$, it is obvious. Suppose that $\ell_{0}^{+}=\ell_{0}^{-}$. First, observe that clearly no point in $\gamma_{i}^{+} \cup \gamma_{i}^{-}, i=1,2$, is a accumulation point of $\alpha_{0}$. So, we have to prove only that there are no accumulation points of $\alpha_{0}$ in $\gamma_{0}^{+} \cup \gamma_{0}^{-}$.

To do this, consider $S_{v}: M \rightarrow M$ the antiholomorphic involution induced by the symmetry of $X(M)$ with respect to the plane $\left\{x_{1}=0\right\}$ (see Theorem 3.17). The normal vector of any fixed point of $S_{v}$ lies in $\left\{x_{1}=0\right\}$. From the order of contact theorem and taking into account that $X(M) \subset \mathscr{E}(\Gamma)$, it is not hard to prove that the normal vector of any point in $\gamma_{0}^{+} \cup \gamma_{0}^{-}$does not lie in $\left\{x_{1}=0\right\}$. In particular, no point of $\gamma_{0}^{+} \cup \gamma_{0}^{-}$is fixed by $S_{v}$. Therefore, if a curve $\alpha \subset \alpha_{0}$ approaches a point lying in $\gamma_{0}^{+} \cup \gamma_{0}^{-}$, no point of $\alpha$ is fixed by $S_{v}$. Hence, the curves $\alpha$ and $S_{v}(\alpha)$ are distinct and mapped under $X$ on the same curve in $\mathbb{R}^{3}$. This contradicts that $\left.X\right|_{M-\partial(M)}$ is injective.

Summarizing, any divergent curve in $\alpha_{0}$ approaches only either $E_{1}$ or $E_{2}$ and only one such curve diverges to the ends. Since there are no compact domains in $M-\partial(M)$ bounded by curves in $\alpha_{0}$ (use the interior maximum principle), we deduce that $\alpha_{0}$ consists of a single regular arc in $M-\partial(M)$ joining $E_{1}$ and $E_{2}$. Furthermore, $\alpha_{0}$ is the fixed point set of $S_{v}$. 
To finish the proof, observe that $p_{3}\left(X\left(\alpha_{0}\right)\right)$ is a closed connected piece of the $x_{2}$-axis. Since the interior maximum principle implies $X(M-\partial(M)) \subset(\mathscr{E}(\Gamma)-\partial(\mathscr{E}(\Gamma))$, it is not hard to prove that

$$
\left.\mathfrak{p}_{3}\right|_{X\left(\alpha_{0}\right)}: X\left(\alpha_{0}\right) \rightarrow\{(0, t, 0) / t>0\}
$$

is not onto. This concludes the proof.

Our purpose is to prove the following result.

THEOREM 3.24. The surface $X(M)$ is symmetric with respect to the plane $\left\{x_{3}=0\right\}$. Furthermore, $M_{+}(0)-\left(\ell_{0}^{+} \cup \ell_{0}^{-}\right)$and $M_{-}(0)-\left(\ell_{0}^{+} \cup \ell_{0}^{-}\right)$are graphs over $\left\{x_{3}=0\right\}$.

PROOF. Let $\mathscr{G}=\{P \in M /|g(P)|=1\}$, and observe that $\mathscr{G}$ is a one dimensional real analytic variety containing $\gamma_{0}^{+} \cup \gamma_{0}^{-}$. This variety consists of the critical points of the projection on the plane $x_{3}=0$.

We label $Q_{i}^{+}=\gamma_{0}^{+} \cap \gamma_{i}^{+}$and $Q_{i}^{-}=\gamma_{0}^{-} \cap \gamma_{i}^{-}, i=1,2$. Taking into account that $X(M) \subset \mathscr{E}(\Gamma)$ and the order of contact theorem, $Q_{i}^{+}$and $Q_{i}^{-}$are not branch points of $g, i=1,2$.

Then, $\gamma_{0}^{+}\left(\gamma_{0}^{-}\right)$is the only curve of $\mathscr{G}$ which approaches $Q_{i}^{+}\left(Q_{i}^{-}\right) ; i=1,2$.

On the other hand, using once again that $X(M) \subset \mathscr{E}(\Gamma)$ and the order of contact theorem, we conclude that $|g(P)| \neq 1$, for $P \in \gamma_{i}^{+} \cup \gamma_{i}^{-}, i=1,2$.

These facts and Theorem 3.12 imply the existence of $t^{\prime}>0$ satisfying $M_{+}\left(t^{\prime}\right) \cap$ $X(\mathscr{G})=M_{+}\left(t^{\prime}\right) \cap\left(\ell_{0}^{+} \cup \ell_{0}^{-}\right)$. Hence, using a symmetric argument, we deduce that $\mathscr{G}$ is compact.

Thus, if $t^{\prime}$ is taken as above, then $\left.\mathfrak{p}_{3}\right|_{M_{+}\left(t^{\prime}\right)-\left(\ell_{0}^{+} \cup \ell_{0}^{-}\right)}$has an injective differential at any point.

CLAIM 3.25. Let $\left.\left.t^{\prime} \in\right] 0,1 / 2\right]$ such that

$$
\left(M_{+}\left(t^{\prime}\right)-\left(\ell_{0}^{+} \cup \ell_{0}^{-}\right)\right) \cap X(\mathscr{G})=\emptyset .
$$

Then $M_{+}\left(t^{\prime}\right)-\left(\ell_{0}^{+} \cup \ell_{0}^{-}\right)$is a graph over the $\left(x_{1}, x_{2}\right)$-plane.

The claim is trivial for $t^{\prime}=1 / 2$. Suppose $t^{\prime}<1 / 2$.

First note that $p_{3}$ is injective along $\left.\left(\ell_{1}^{+} \cup \ell_{1}^{-} \cup \delta_{t^{\prime}}\right)-\left(\ell_{0}^{+} \cup \ell_{0}^{-}\right)\right)$.

Define $v=\mathfrak{p}_{3}\left(\delta_{t^{\prime}}\right)$ and $\mathscr{E}(\Gamma)_{0}=\mathfrak{p}_{3}(\mathscr{E}(\Gamma))$. As $v-\left(\ell_{0}^{+} \cup \ell_{0}^{-}\right)$has no selfintersections, then $\mathscr{E}(\Gamma)_{0}-v$ has two connected components $\Upsilon_{1}$ and $\Upsilon_{2}$. Up to relabeling, we suppose that $\Upsilon_{1}$ is the bounded component, and so $\Upsilon_{2}$ is the unbounded one. See Figure 11.

Since $X(\partial(M))=\Gamma, X(M-\partial(M)) \cap \partial(\mathscr{E}(\Gamma))=\emptyset, X(M) \subset \mathscr{E}(\Gamma)$ and $\left(M_{+}\left(t^{\prime}\right)-\right.$ $\left.\left(\ell_{0}^{+} \cup \ell_{0}^{-}\right)\right) \cap X(\mathscr{G})=\emptyset$, then $\left.\mathfrak{p}_{3}\right|_{M_{+}\left(t^{\prime}\right)-\left(\ell_{0}^{+} \cup \ell_{0}^{-} \cup \delta_{r^{\prime}}\right)}$ is a local diffeomorphism onto its 


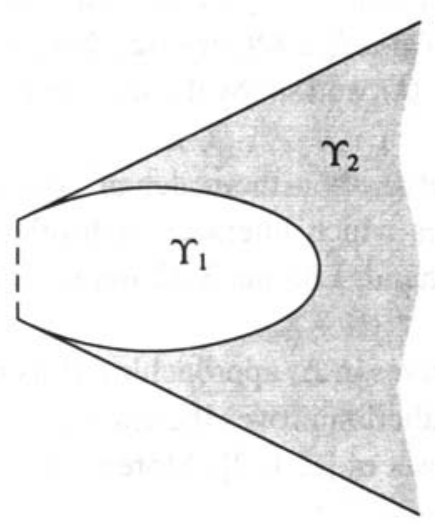

FIGURE 11. $\mathscr{E}(\Gamma)_{0}-v$ has two connected components $\Upsilon_{1}$ and $\Upsilon_{2}$.

image in $\mathscr{E}(\Gamma)_{0}$. Hence, $\Upsilon=\mathfrak{p}_{3}\left(M_{+}\left(t^{\prime}\right)-\left(\ell_{0}^{+} \cup \ell_{0}^{-} \cup \delta_{t^{\prime}}\right)\right)$ is an open subset of $\mathscr{E}(\Gamma)_{0}$. Moreover, $\left.\mathfrak{p}_{3}\right|_{X(M)}$ is proper, and so $\Upsilon \cap \Upsilon_{i}$ is a closed subset of $\Upsilon_{i}, i=1,2$. Therefore, either $\Upsilon_{i} \subset \Upsilon$ or $\Upsilon \cap \Upsilon_{i}=\emptyset, i=1,2$.

From Theorem 3.17 and Lemma 3.23, $\Upsilon \cap \Upsilon_{1}=\emptyset$, and so $\Upsilon_{2} \subset \Upsilon$, which easily implies $\Upsilon=\Upsilon_{2}$.

All these arguments give easily that $\left.\mathfrak{p}_{3}\right|_{M_{+}\left(t^{\prime}\right)-\left(\ell_{0}^{+} \cup \ell_{0}^{-}\right)}$is a proper local homeomorphism onto $\Upsilon \cup v$, that is $\left.\mathfrak{p}_{3}\right|_{M_{+}\left(t^{\prime}\right)-\left(\ell_{0}^{+} \cup \ell_{0}^{-}\right)}$is a covering map.

As $\Upsilon \cup v$ is simply connected, then $\left.\mathfrak{p}_{3}\right|_{M_{+}\left(t^{\prime}\right)-\left(\ell_{0}^{+} \cup \ell_{0}^{-}\right)}$is a homeomorphism, that is, $M_{+}\left(t^{\prime}\right)-\left(\ell_{0}^{+} \cup \ell_{0}^{-}\right)$is a graph over $\left\{x_{3}=0\right\}$. This proves the claim.

Define now the set

$$
\begin{aligned}
\mathscr{I}_{2}= & \left\{t \in \left[0,1 / 2\left[/ M_{+}(t)-\left(\ell_{0}^{+} \cup \ell_{0}^{-}\right) \text {is a graph over the }\left(x_{1}, x_{2}\right)\right. \text {-plane }\right.\right. \\
& \text { and } \left.M_{+}^{*}(t) \succeq M_{-}(t)\right\} .
\end{aligned}
$$

\section{CLAIM 3.26. $\mathscr{I}_{2}$ is not empty.}

Let $t^{\prime}$ be satisfying $\left(M_{+}\left(t^{\prime}\right)-\left(\ell_{0}^{+} \cup \ell_{0}^{-}\right)\right) \cap X(\mathscr{G})=\emptyset$, and take $t \in\left[\left(2 t^{\prime}+1\right) / 4,1 / 2[\right.$. From Claim 3.25, $M_{+}^{*}(t)$ is a graph over the plane $x_{3}=0$, and so it is obvious that $M_{+}^{*}(t) \succeq M_{-}(t)$. Thus, $t \in \mathscr{I}_{2}$, as we want to prove.

For any $t \in] 0,1 / 2\left[\right.$, label $\Delta_{t}=M_{+}^{*}(t) \cap M_{-}(t)$. Observe that Theorem 3.12 implies that no curve in $\Delta_{t}$ diverges to $E_{i}, i=1,2$, that is, $\Delta_{t}$ is compact, $\left.t \in\right] 0,1 / 2$.

Furthermore, for each $t \in] 0,1 / 2$, we write $P_{1}^{t}, P_{2}^{t}$ for the only two points in the subset $X^{-1}\left(\delta_{\ell} \cap\left(\ell_{0}^{+} \cup \ell_{0}^{-}\right)\right)$. With this notation we can assert:

ClaIM 3.27. If $t \in \mathscr{I}_{2}, t>0$, then $P_{i}^{t}$ is not a branch point of $g, i=1,2$. 
Consider $t \in \mathscr{I}_{2}, t>0$. As $M_{+}^{*}(t) \succeq M_{-}(t),(X(M)-\Gamma) \subset(\mathscr{E}(\Gamma)-\partial(\mathscr{E}(\Gamma))$ and $t>0$, then $\Delta_{t} \subset \delta_{t} \cup \ell_{0}^{+} \cup \ell_{0}^{-}$. Otherwise, there would be an interior contact point between $M_{+}^{*}(t)$ and $M_{-}(t)$, and so, by the interior maximum principle, $M_{+}^{*}(t)=$ $M_{-}(t)$, which is absurd.

If $P_{i}^{t}$ is a branch point of $g$, then the order of contact theorem implies that $\Delta_{t}$ contains at least three curves which intersect each other at this point and forming equal angles. On the other hand, Lemma 3.22 implies that $X(M)$ and $\left\{x_{3}=t\right\}$ are transverse.

So, at least one of these curves in $\Delta_{t}$ approaching $P_{i}^{t}$ is not contained in $\delta_{t} \cup \ell_{0}^{+} \cup \ell_{0}^{-}$, which is absurd. This contradiction proves the claim.

Clearly $\mathscr{I}_{2}$ is a closed subset of $[0,1 / 2[$. Moreover,

CLAIM 3.28. The minimum of $\mathscr{I}_{2}$ is 0 .

Let $t_{0} \in \mathscr{I}_{2}$ and suppose that $t_{0}>0$. Since $M_{+}\left(t_{0}\right)-\left(\ell_{0}^{+} \cup \ell_{0}^{-}\right)$is a graph over the plane $\left\{x_{3}=0\right\}$ and $M_{+}^{*}\left(t_{0}\right) \succeq M_{-}\left(t_{0}\right)$, then $M_{+}\left(t_{0}\right) \cap X(\mathscr{G}) \subset \ell_{0}^{+} \cup \ell_{0}^{-}$. Otherwise, $\delta_{t_{0}}-\left\{X\left(P_{1}^{t_{0}}\right), X\left(P_{2}^{t_{0}}\right)\right\}$ would contain a point of $X(\mathscr{G})$, and so the maximum principle at the boundary would imply that $M_{+}^{*}\left(t_{0}\right)=M_{-}\left(t_{0}\right)$, which is absurd.

Hence, by Claim 3.27 , the points $P_{i}^{t_{0}}$ are not ramification points of $g$. Using that $X(\mathscr{G})$ is compact, there exists $\epsilon>0$ small enough such that $t_{0}-\epsilon>0$ and $M_{+}\left(t_{0}-\epsilon\right) \cap X(\mathscr{G}) \subset\left(\ell_{0}^{+} \cup \ell_{0}^{-}\right)$. From Claim 3.25, $M_{+}\left(t_{0}-\epsilon\right)-\left(\ell_{0}^{+} \cup \ell_{0}^{-}\right)$is a graph over $\left\{x_{3}=0\right\}$.

Therefore,

$$
\Delta_{t}-\left(\ell_{0}^{+} \cup \ell_{0}^{-} \cup \delta_{t}\right) \subset M_{-}\left(t_{0}-\epsilon\right), \quad \forall t \in\left[t_{0}-\epsilon, 1 / 2[.\right.
$$

We are going to prove that $\Delta_{t} \subset\left(\delta_{t} \cup \ell_{0}^{+} \cup \ell_{0}^{-}\right), t \in\left[t_{0}-\epsilon, t_{0}\right]$.

As we have said before, $t_{0} \in \mathscr{I}_{2}$ yields $\Delta_{t_{0}}-\left(\delta_{t_{0}} \cup \ell_{0}^{+} \cup \ell_{0}^{-}\right)=\emptyset$.

Let $t^{\prime} \in\left[t_{0}-\epsilon, t_{0}\left[\right.\right.$ and write $t_{1}=\operatorname{Infimum}\left\{t \in\left[t^{\prime}, t_{0}\right] / \Delta_{t}-\left(\delta_{t} \cup \ell_{0}^{+} \cup \ell_{0}^{-}\right)=\emptyset\right\}$. Notice that $\Delta_{t_{1}}-\left(\delta_{t_{1}} \cup \ell_{0}^{+} \cup \ell_{0}^{-}\right)=\emptyset$. Otherwise, and taking into account that $(X(M)-\Gamma) \subset\left(\mathscr{E}(\Gamma)-\partial(\mathscr{E}(\Gamma))\right.$, we could see that $M_{+}^{*}\left(t_{1}\right)$ and $M_{-}\left(t_{1}\right)$ would have an interior point of contact in $M_{-}\left(t_{0}-\epsilon\right)$. Thus, the interior maximum principle would imply $M_{+}^{*}\left(t_{1}\right)=M_{-}\left(t_{1}\right)$, which is a contradiction (recall that $\left.t_{1}>0\right)$.

In particular, the order of contact theorem gives that $M_{+}^{*}\left(t_{1}\right)$ and $M_{-}\left(t_{1}\right)$ are transverse along $\Delta_{t_{1}}-\left\{X\left(P_{1}^{t_{1}}\right), X\left(P_{2}^{t_{1}}\right)\right\} \subset \delta_{t_{1}} \cup \ell_{0}^{+} \cup \ell_{0}^{-}$, and $\left\{X\left(P_{1}^{t_{1}}\right), X\left(P_{2}^{t_{1}}\right)\right\}$ are points of ordinary contact. In case $\ell_{0}^{+}=\ell_{0}^{-}$, this means that the four sheets of $M_{-}\left(t_{1}\right) \cup M_{+}^{*}\left(t_{1}\right)$ are transverse at any point of $\ell_{0}^{+} \cap \Delta_{t_{1}}=\ell_{0}^{-} \cap \Delta_{t_{1}}$, except at the point $X\left(P_{1}^{t_{1}}\right)=X\left(P_{2}^{t_{1}}\right)$, where just two couples of sheets are tangent.

If $t_{1}>t^{\prime}$, then the definition of $t_{1}$ and (11) imply the existence of sequences $\left\{t_{n}\right\}_{n \in \mathrm{N}}$ in $] t^{\prime}, t_{1}\left[,\left\{p_{n}\right\}_{n \in \mathbb{N}}\right.$ in $M_{+}\left(t_{n}\right)-\left(\delta_{t_{n}} \cup \ell_{0}^{+} \cup \ell_{0}^{-}\right)$and $\left\{q_{n}\right\}_{n \in \mathrm{N}}$ in $M_{-}\left(t_{0}-\epsilon\right)-\left(\delta_{t_{n}} \cup \ell_{0}^{+} \cup \ell_{0}^{-}\right)$ such that: $\lim _{n \rightarrow \infty} t_{n}=t_{1}$ and $q_{n}=p_{n}^{*}\left(t_{n}\right)$. Without loss of generality, we can suppose 
that $\left\{p_{n}\right\}_{n \in N}$ and $\left\{q_{n}\right\}_{n \in N}$ converge to $p_{0} \in M_{+}\left(t_{1}\right)$ and $q_{0} \in M_{-}\left(t_{0}-\epsilon\right)$. Since $q_{0}=p_{0}^{*}\left(t_{1}\right)$, we have $q_{0} \in \Delta_{t_{1}} \cap M_{-}\left(t_{0}-\epsilon\right)$. Therefore, and taking into account that $t_{0}-\epsilon<t_{1}$, we get $q_{0} \in\left(\left(\ell_{0}^{+} \cup \ell_{0}^{-}\right)-\left\{X\left(P_{1}^{f_{1}}\right), X\left(P_{2}^{t_{1}}\right)\right\}\right)$. This obviously contradicts that $M_{+}^{*}\left(t_{1}\right)$ and $M_{-}\left(t_{1}\right)$ are transverse at $q_{0}$.

Hence, we deduce that $t_{1}=t^{\prime}$, and since $t^{\prime}$ is an arbitrary point of $\left[t_{0}-\epsilon, t_{0}[\right.$, we have

$$
\left.\left.\Delta_{t} \subset\left(\delta_{t} \cup \ell_{0}^{+} \cup \ell_{0}^{-}\right), \quad t \in\right] t_{0}-\epsilon, t_{0}\right] .
$$

Since $M_{+}^{*}\left(t_{0}\right) \succeq M_{-}\left(t_{0}\right)$ and $X(M) \subset \mathscr{E}(\Gamma)$, a continuity argument gives $M_{+}^{*}(t) \succeq$ $M_{-}(t), t \in\left[t_{0}-\epsilon, t_{0}\right]$. This proves that $\left[t_{0}-\epsilon, t_{0}\right] \subset \mathscr{I}_{2}$, and so the claim.

Last claim implies that $M_{+}(0)-\left(\ell_{0}^{+} \cup \ell_{0}^{-}\right)$is a graph over $\left\{x_{3}=0\right\}$ and $M_{+}^{*}(0) \succeq$ $M_{-}(0)$. If we repeat symmetrically the same arguments from below, we get that $M_{-}(0)-\left(\ell_{0}^{+} \cup \ell_{0}^{-}\right)$is a graph over $\left\{x_{3}=0\right\}$ and $M_{-}^{*}(0) \succeq M_{+}(0)$. This concludes the proof.

This theorem has two interesting consequences.

COROLLARY 3.29. There are only two branch points $R_{0}^{+} \in \gamma^{+}$and $R_{0}^{-} \in \gamma^{-}$ of $g$ along $\gamma_{0}^{+} \cup \gamma_{0}^{-}$. Furthermore, $g$ has multiplicity two at these points and $X\left(R_{0}^{+}\right), X\left(R_{0}^{-}\right) \in \delta_{0}$.

PROOF. Theorem 3.24 implies that $\mathscr{G}=X^{-1}\left(\delta_{0}\right) \cup \gamma_{0}^{+} \cup \gamma_{0}^{-}$. If we label $R_{0}^{+}=$ $\gamma_{0}^{+} \cap X^{-1}\left(\delta_{0}\right)$ and $R_{0}^{-}=\gamma_{0}^{-} \cap X^{-1}\left(\delta_{0}\right)$, then elementary properties of meromorphic functions give that $R_{0}^{+}$and $R_{0}^{-}$are branch points of $g$ with multiplicity two.

COROLLARY 3.30. The limit normal vectors at the ends are opposite.

In what follows and without loss of generality, we assume that

$$
g\left(E_{1}\right)=0, \quad g\left(E_{2}\right)=\infty .
$$

3.3. The geometrical uniqueness In this subsection, we determine the conformal structure and Weierstrass data associated to the minimal immersion $X: M \rightarrow \mathbb{R}^{3}$ described at the beginning of this section.

Define

$$
\begin{array}{ll}
\left.\left.r_{1}^{+}=\left\{\lambda e^{-\theta i / 2}: \lambda \in\right] 0,1\right]\right\}, & \left.\left.r_{1}^{-}=\left\{-\lambda e^{\theta i / 2}: \lambda \in\right] 0,1\right]\right\}, \\
r_{2}^{+}=\left\{\lambda e^{-\theta i / 2}: \lambda \in[1,+\infty[\},\right. & r_{2}^{-}=\left\{-\lambda e^{\theta i / 2}: \lambda \in[1,+\infty[\} .\right.
\end{array}
$$

Recall that $g\left(E_{1}\right)=0=(1 / g)\left(E_{2}\right)$, and as before, $Q_{j}^{+}=\gamma_{0}^{+} \cap \gamma_{j}^{+}, Q_{j}^{-}=\gamma_{0}^{-} \cap \gamma_{j}^{-}$, $j=1,2$. Since $X(M) \subset \mathscr{E}(\Gamma)$, it is not hard to see that $g(P) \neq g\left(Q_{j}^{+}\right)$(respectively 


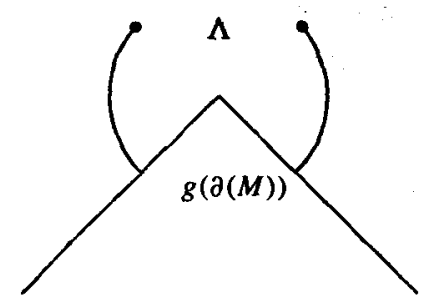

FIGURE 12. The region $\Lambda$ and the curve $g(\partial(M))$.

$\left.g(P) \neq g\left(Q_{j}^{-}\right)\right)$, for $P \in\left(\gamma_{j}^{+}-\left\{Q_{j}^{+}\right\}\right)$(for $P \in\left(\gamma_{j}^{-}-\left\{Q_{j}^{-}\right\}\right)$). Then, it is now clear that $g\left(\gamma_{j}^{+}\right)=r_{j}^{+}, g\left(\gamma_{j}^{-}\right)=r_{j}^{-}, j=1,2$.

On the other hand, from Corollary 3.29 , we have that $\left.g\right|_{\left[Q_{j}^{+}, R_{0}^{+}\right]}$and $\left.g\right|_{\left[Q_{j}^{-}, R_{0}^{-}\right]}$are injective, $j=1,2$, and Theorem 3.24 yields $g\left(\gamma_{0}^{+}\right)=g\left(\left[Q_{j}^{+}, R_{0}^{+}\right]\right)$and $g\left(\gamma_{0}^{-}\right)=$ $g\left(\left[Q_{j}^{-}, R_{0}^{-}\right]\right), j=1,2$. Note that $g\left(Q_{j}^{+}\right)=e^{-i \theta / 2}$ and $g\left(Q_{j}^{-}\right)=e^{i \theta / 2}$. Moreover, $X(M) \subset \mathscr{E}(\Gamma)$ implies that:

- $g\left(R_{0}^{+}\right)=e^{-t_{0} i}, g\left(R_{0}^{-}\right)=-e^{t_{0} i}$, where $\left.t_{0} \in\right]-\pi / 2, \theta / 2[$.

- $g(P) \neq \pm i$, for $P \in \gamma_{0}^{+} \cup \gamma_{0}^{-}$.

Then it is easy to conclude that $g\left(\gamma_{0}^{+}\right)=r_{0}^{+}$and $g\left(\gamma_{0}^{-}\right)=r_{0}^{-}$, where $r_{0}^{+}$and $r_{0}^{-}$are given as

$$
r_{0}^{+}=\left\{e^{i t}: t \in\left[-\theta / 2,-t_{0}\right]\right\}, \quad r_{0}^{+}=\left\{-e^{i t}: t \in\left[t_{0}, \theta / 2\right]\right\}
$$

Summarizing, we deduce that $g(\partial(M))=\bigcup_{j=0}^{2}\left(r_{j}^{+} \cup r_{j}^{-}\right)$.

Let $\Lambda$ denote the connected component of $\mathbb{C}-(g(\partial(M)) \cup\{0\})$ containing $i=\sqrt{-1}$. Note that $\left(r_{0}^{+} \cup r_{0}^{-}\right) \subset \partial(\Lambda)$. See Figure 12 .

The following lemma is a consequence of the information included in Subsection 3.1 and Subsection 3.2 .

LEMMA 3.31. The map $g: M \rightarrow \mathbb{C}$ satisfies:

1. $g(M-\partial(M))=\Lambda$ and $\left.g\right|_{M-\partial(M)}: M-\partial(M) \rightarrow \Lambda$ is a biholomorphism.

2. $g\left(\gamma_{j}^{+}\right)=r_{j}^{+}, g\left(\gamma_{j}^{-}\right)=r_{j}^{-}, j=1,2$. Furthermore, $\left.g\right|_{\gamma_{j}^{+}},\left.g\right|_{\gamma_{j}^{-}}$are injective, $j=1,2$.

3. $g\left(\gamma_{0}^{+}\right)=r_{0}^{+}$and $g\left(\gamma_{0}^{-}\right)=r_{0}^{-}$. Moreover, $\left.g\right|_{\left[Q_{j}^{+}, R_{0}^{+}\right]},\left.g\right|_{\left.\mathcal{Q}_{j}^{-}, R_{0}^{-}\right]}$are injective, $j=1,2$. 
PROOF. To see that $g(M-\partial(M))=\Lambda$, let us observe that $g(M-\partial(M))$ is a connected component of $\mathbb{C}-(g(\partial(M)) \cup\{0\})$. From Lemma 3.22, it is obvious that $g(M-\partial(M))$ is an open subset of $\mathbb{C}^{*}$ (holomorphic non constant functions are open). Moreover, as $\bar{M}=M \cup\left\{E_{1}, E_{2}\right\}$ is compact, then Theorem 3.12 gives that $g(\bar{M})$ is a closed subset of $\overline{\mathbb{C}}$. In particular, the set $W=g(M-\partial(M)) \cap(\mathbb{C}-(g(\partial(M)) \cup\{0\})=$ $g(\bar{M}) \cap(\mathbb{C}-(g(\partial(M)) \cup\{0\})$ is a closed subset of $\mathbb{C}-(g(\partial(M)) \cup\{0\})$. Therefore, either $W=\mathbb{C}-(g(\partial(M)) \cup\{0\})$ or $W$ is a connected component of $\mathbb{C}-(g(\partial(M)) \cup\{0\})$. Since there are points $\omega \in g(M-\partial(M))$ such that $-\omega \notin g(M-\partial(M)$ ) (see Lemma 3.5), then $W$ is a connected component of $\mathbb{C}-(g(\partial(M)) \cup\{0\})$. Taking into account that the points in $r_{0}^{+}, r_{0}^{-}$lie in the boundary of $W$, we easily deduce that $W=\Lambda$. So, $g(M-\partial(M))=\Lambda$.

To finish the proof of 1 , define $\gamma=\partial(\bar{M})$. Since $\bar{M}$ is conformally a closed disk with piecewise analytic boundary (see Theorem 3.8), then $\gamma$ is a piecewise analytic curve homeomorphic to $\mathbb{S}^{1}$. Moreover, as $\left.g\right|_{M}$ has no poles and $g$ extends continuously to $\bar{M}$, then we know that for any $\omega \in \Lambda=g(M-\partial(M))$,

$$
\sharp\left(g^{-1}(\omega)\right)=\frac{1}{2 \pi i} \int_{\gamma} \frac{d g}{g-\omega} \in \mathbb{Z} .
$$

Thus, if we define $f: \Lambda \rightarrow \mathbb{Z}$ by $f(\omega)=\sharp\left(g^{-1}(\omega)\right)$, the function $f$ is continuous on $\Lambda$, and so it is constant. Since there exists $\omega \in \Lambda$ such that $f(\omega)=1$ (see Lemma 3.5), we deduce that $f=1$, that is, $g: M-\partial(M) \rightarrow \Lambda$ is a biholomorphism, which proves 1 .

To prove 2, use 1 to check that $\left.g\right|_{\gamma_{1}^{+}}$has no branch points, and the same occurs for $\gamma_{1}^{-}, \gamma_{2}^{+}$and $\gamma_{2}^{-}$. The remaining of 2 , and 3 were proved at the beginning of this subsection.

At this point we are able to prove the principal result of this section.

THEOREM 3.32. The immersion $X: M \rightarrow \mathbb{R}^{3}$ is, up to a horizontal translation, one of the minimal surfaces $X_{\theta r}$ described in (7), for a suitable $\left.r \in\right]-1, r_{\theta}$ ].

In particular, $\ell_{0}^{+}=\ell_{0}^{-}$if and only if $r=r_{\theta}$.

PROOF. Let $M^{\prime}$ denote the Riemann surface obtained from $M$ by successive Schwarz reflections about $\gamma_{j}^{+}$and $\gamma_{j}^{-}, j=1,2$. Note that $\partial\left(M^{\prime}\right) \neq \emptyset$.

Let $X^{\prime}: M^{\prime} \rightarrow \mathbb{R}^{3}$ be the corresponding minimal immersion, and label $g^{\prime}$ as its Gauss map. Obviously, $\left.g^{\prime}\right|_{M}=g$. Observe that $X^{\prime}\left(M^{\prime}\right)$ is invariant under the translation $T$ defined as the composition of the 180 degrees rotation about $\ell_{1}^{+}$and $\ell_{2}^{+}$. The translation $T$ induces a holomorphic transformation without fixed points $T^{\prime}$ : $M^{\prime} \rightarrow M^{\prime}$. We denote $M_{0}=M^{\prime} /\left\langle T^{\prime}\right\rangle$ and $p_{0}: M^{\prime} \rightarrow M_{0}$ as the natural projection. Write $X_{0}: M_{0} \rightarrow \mathbb{R}^{3} /\langle T\rangle$ as the minimal immersion satisfying $X_{0} \circ p_{0}=X^{\prime}$. It is clear that $\partial\left(M_{0}\right) \neq \emptyset$. 
Clearly, $\left.p_{0}\right|_{M}: M \rightarrow p_{0}(M)$ is a biholomorphism. In what follows, we identify $M$ and $p_{0}(M)$, and so, we consider $M$ as a subset of $M_{0}$.

The symmetries of $X(M)$ deduced in Subsection 3.2 together with the 180 degrees rotations about the horizontal lines, generate a group of symmetries of $X^{\prime}\left(M^{\prime}\right)$ which contains a rotation about a straight line parallel to the $x_{3}$-axis by angle $\theta$, followed by a symmetry with respect the plane $x_{3}=1 / 2$. We call this symmetry $J$.

We denote by $S_{v}$ and $S_{h}$ the antiholomorphic involutions on $M_{0}$ induced by the symmetries with respect to the planes $x_{1}=0$ and $x_{3}=0$, respectively. Write $L^{+}$and $L^{-}$for the antiholomorphic involutions on $M_{0}$ induced by the 180 degrees rotations about the straight lines containing $\ell_{2}^{+}$and $\ell_{2}^{-}$, respectively.

Note that $J$ is the composition of the 180 degrees rotation about the straight line containing $\ell_{2}^{+}$and the symmetry with respect to the plane $x_{1}=0$, and so it induces on $M_{0}$ the holomorphic transformation $J_{0}=S_{v} \circ L^{+}$.

We denote by $g_{0}: M_{0} \rightarrow \overline{\mathbb{C}}$ the Gauss map of $X_{0}$, and observe that, up to natural identifications, $\left.g_{0}\right|_{M}=g$.

As $M$ is simply connected, $\log (g)$ has a well defined branch on $M$, and so the same occurs for $g^{n}$, where

$$
n=\frac{2 \pi}{\pi+\theta} .
$$

We choose the branch of $\log (g)$ in such a way that $\arg (\log (g(P)))=0$, whenever $g(P) \in \mathbb{R}^{+}$. This choice is possible as consequence of Lemma 3.31. Furthermore, if $P \in \partial(M)-\left(\gamma_{0}^{+} \cup \gamma_{0}^{-}\right)$, then taking into account that $g_{0} \circ J_{0}=e^{2 \pi i / n} g_{0}$ and Lemma 3.31, we get $g^{n}(P)=g^{n}\left(J_{0}(P)\right)$.

On the other hand, it is clear that $M_{0}=\bigcup_{j=1}^{\infty} J_{0}^{j}(M)$, and recall that $\left.g_{0}\right|_{M}=g$. As above, we can define suitable branches $h_{j}$ of $\left(\left.g_{0}\right|_{J_{0}^{j}(M)}\right)^{n}, j \in \mathbb{N}$, in such a way that

- $h_{0}=g^{n}$ is the above fixed branch .

- If $P \in J_{0}^{j}\left(\partial(M)-\left(\gamma_{0}^{+} \cup \gamma_{0}^{-}\right)\right)$, then $h_{j}(P)=h_{j}\left(J_{0}(P)\right)$.

- If $P \in J_{0}^{j}(M) \cap J_{0}^{j+1}(M)$, then $h_{j}(P)=h_{j+1}(P)$.

We easily deduce that $g_{0}^{n}$ has a well defined branch on $M_{0}$ satisfying $g_{0}^{n} \circ J_{0}=g_{0}^{n}$ and $\left.g_{0}^{n}\right|_{M}=g^{n}$.

Hence, if we write $\zeta: M_{0} \rightarrow M_{0} /\left\langle J_{0}\right\rangle$ for the natural projection, then there exists a holomorphic transformation $f_{0}: M_{0} /\left\langle J_{0}\right\rangle \rightarrow \mathbb{C}^{*}$, satisfying $g_{0}^{n}=f_{0} \circ \zeta$.

Taking into account our choice of the branches, we have:

- $g_{0}^{n}\left(\gamma_{1}^{+} \cup \gamma_{2}^{+}\right)=g_{0}^{n}\left(\gamma_{1}^{-} \cup \gamma_{2}^{-}\right)=\left\{-\lambda e^{n \pi i / 2} / \lambda>0\right\}$.

- $\left.g_{0}^{n}\right|_{\left[Q_{j}^{+}, R_{0}^{+}\right\}}$and $\left.g_{0}^{n}\right|_{\left[Q_{j}^{-}, R_{0}^{-}\right]}$are injective, $j=1,2$. Furthermore, $g_{0}^{n}\left(\left[Q_{j}^{+}, R_{0}^{+}\right]\right)=$ $l^{\prime}$ and $g_{0}^{n}\left(\left[Q_{j}^{-}, R_{0}^{-}\right]\right)=l^{\prime \prime}, j=1,2$, where

$$
\begin{aligned}
l^{\prime} & =\left\{e^{-t i} / t \in\left[n \pi / 2-\pi,-n t_{0}\right]\right\}, \\
l^{\prime \prime} & =\left\{e^{-t i} / t \in\left[-2 \pi+n \pi+n t_{0}, n \pi / 2-\pi\right]\right\} .
\end{aligned}
$$


- $g_{0}^{n}\left(M_{0}-\partial\left(M_{0}\right)\right)=f_{0}\left(\left(M_{0} /\left\langle J_{0}\right\rangle-\partial\left(M_{0} /\left\langle J_{0}\right)\right)\right)=\mathbb{C}^{*}-l\right.$, where $l=l^{\prime} \cup l^{\prime \prime}$. Furthermore, from Lemma 3.31, it is not hard to prove that

$$
\left.f_{0}\right|_{M_{0} /\left(J_{0}\right)-\partial\left(M_{0} /\left(J_{0}\right)\right)}: M_{0} /\left(J_{0}\right\rangle-\partial\left(M_{0} /\left\langle J_{0}\right\rangle\right) \rightarrow C^{*}-l
$$

is a biholomorphism.

Let $\mathscr{S}$ be the Riemann surface

$$
\mathscr{S}=\left\{(y, v) \in \mathbb{C}^{*} \times \mathbb{C} / v^{2}=\left(y-e^{-n t_{0} i}\right)\left(y-e^{n\left(t_{0}+\pi\right) i}\right)\right\} .
$$

The set $y^{-1}\left(\mathbb{C}^{*}-l\right)$ has two connected components in $\mathscr{S}$, and both of them are biholomorphic to $\mathbb{C}^{*}-l$. We label the closure of one of these connected components by $\Lambda_{0}$.

From above arguments, and in a natural way, we can define a map

$$
\hat{f_{0}}: M_{0} /\left\langle J_{0}\right\rangle \rightarrow \Lambda_{0}
$$

such that $y \circ \hat{f_{0}}=f_{0}$. It is not hard to see that $\hat{f_{0}}$ is a biholomorphism. In what follows and for simplicity, we put $f_{0}=\hat{f_{0}}$.

Note that $f_{0}\left(\partial\left(M_{0} /\left(J_{0}\right\rangle\right)\right)=\partial\left(\Lambda_{0}\right)=l^{+} \cup l^{-}$, where $l^{+}$and $l^{-}$are the two lifts of $l$ to $\mathscr{S}$.

It is clear that

$$
\xi=f_{0} \circ \zeta: M_{0} \rightarrow \Lambda_{0}
$$

is an unbranched cyclic covering.

We want to describe in a more precise way the Riemann surface $M_{0}$ and the Weierstrass data of $X_{0}$. This step is fundamental in order to obtain a model of the complex structure of $M$ and Weierstrass data of $X$.

To do this, we are going to determine the covering $\xi$.

Remember that $J_{0}$ is the conformal transformation on $M_{0}$ induced by the counterclockwise rotation about a straight line parallel to the $x_{3}$-axis by an angle of $\theta$ followed by a symmetry with respect to the plane $x_{3}=1 / 2$ on $X^{\prime}\left(M^{\prime}\right)$. Let $c_{1}$ and $c_{2}$ be counterclockwise circuits in $\Lambda_{0}$ around zero and $z^{-1}(l)$, respectively, and $\tilde{c}_{i}$, $i=1,2$, their lifts to $M_{0}$. The end points of $\tilde{c_{i}}$ will differ by a deck transformation of the form $J_{0}{ }^{k_{i}}, k_{i} \in \mathbb{Z}, i=1,2$. The choice of $J_{0}$ implies that $k_{1}=1$ and $k_{2}=0$. The numbers $k_{1}$ and $k_{2}$ determine the induced map from $\Pi_{1}\left(\Lambda_{0}\right)$ onto $\mathbb{Z}_{\text {ord }\left(J_{0}\right)}$ whose kernel corresponds to $\xi_{*}\left(\Pi_{1}\left(M_{0}\right)\right)$, where ord $\left(J_{0}\right)$ is the order of the automorphism $J_{0}$ (that is, the number of sheets of the cyclic covering $\xi$ ). As usual, $\mathbb{Z}_{\infty}=\mathbb{Z}$.

Any cyclic covering of $\Lambda_{0}$ is equivalent to $\xi$ if the associated representation has the same kernel.

At this point, we distinguish two cases: ord $\left(J_{0}\right)<\infty$ and ord $\left(J_{0}\right)=\infty$. 
Case 1: ord $\left(J_{0}\right)<\infty$. Note that ord $\left(J_{0}\right)<\infty$ if and only if $\left.n \in\right] 1,2[\cap \mathbb{Q}$. Label $m=\pi / \theta=n /(2-n)$ and observe that $m \in[1,+\infty[\cap \mathbb{Q}$. Write $m=p / q$, where $p, q \in \mathbb{N}$ and $\operatorname{gcd}(p, q)=1$. Since $J_{0}$ is a rotation by angle $\theta$ followed by a symmetry, then ord $\left(J_{0}\right)=2 p$.

Let $\widetilde{\mathscr{S}}$ be the covering Riemann surface of $\mathscr{S}$ where the function $u=y^{1 /(2 p)}$ is well defined and where

$$
\mathfrak{p}: \tilde{\mathscr{S}} \rightarrow \mathscr{S}
$$

is the natural projection. This covering is cyclic with group of deck transformations of order $2 p$ and generated by

$$
\tilde{J}_{0}((y, v), u)=\left((y, v), e^{\pi i / p} u\right) .
$$

If we define $\mathscr{M}_{0}=\mathfrak{p}^{-1}\left(\Lambda_{0}\right)$ and take into account the above arguments, we infer that the cyclic covering

$$
\left.\mathfrak{p}\right|_{\mathscr{M}_{0}}: \mathscr{M}_{0} \rightarrow \Lambda_{0}
$$

is conformally equivalent to $\xi$.

Up to this biholomorphism, we may assume $\mathscr{M}_{0}=M_{0}, \widetilde{J}_{0}=J_{0}$. The functions $y$ and $v$, up to composition with the covering map $\mathfrak{p}$, are meromorphic functions on $\boldsymbol{M}_{\mathbf{0}}$. Taking into account that $y=g_{0}^{n}$, we deduce also that, up to a suitable choice of the initial condition of the biholomorphism, $g_{0}=u^{p+q}$.

Summarizing, we have fixed the conformal structure of $M_{0}$ and the meromorphic function $g_{0}$.

Label $\left(\phi_{1}^{0}, \phi_{2}^{0}, \phi_{3}^{0}\right)$ as the holomorphic 1-forms of the Weierstrass data of $X_{0}$. Let us determine $\phi_{3}^{0}$.

It is clear that $J_{0}^{*}\left(\phi_{3}^{0}\right)=-\phi_{3}^{0}$, and so the meromorphic function $f_{3}=\phi_{3}^{0} / d y$ satisfies $f_{3} \circ J_{0}=-f_{3}$. This implies that $f_{3}=u^{p} h_{3}$, where $h_{3}$ is the lift to $M_{0}$ of a meromorphic function on $\Lambda_{0}$.

As $X_{0}\left(\partial\left(M_{0}\right)\right)$ consists of the projection to $\mathbb{R}^{3} /\langle T\rangle$ of a set of straight lines in $\mathbb{R}^{3}$ parallel to the $x_{3}$-axis, it is easy to check that if ${ }_{3}(P) y(P) \in \mathbb{R}$, for any $P \in \partial\left(M_{0}\right)$. Furthermore, the antiholomorphic involution $S_{h}$ induced by the horizontal symmetry satisfies $g_{0} \circ S_{h}=1 / \overline{g_{0}}$ and $S_{h}^{*}\left(\phi_{3}^{0}\right)=-\overline{\phi_{3}^{0}}$. This implies that $y \circ S_{h}=1 / \bar{y}$, $v \circ S_{h}=e^{n \pi i / 2} \bar{v} / \bar{y}$ and $\left(y f_{3}\right) \circ S_{h}=\overline{y f_{3}}$.

Thus, given $P \in \partial\left(M_{0}\right)$, we have $\left(y f_{3}\right)\left(S_{h}(P)\right)=-\left(y f_{3}\right)(P)$.

Any connected component $\widetilde{l}$ of $\partial\left(M_{0}\right)$ is a homeomorphic copy of $l^{+} \cup l^{-}$, and since $v\left(S_{h}(P)\right)=-v(P), \forall P \in \partial\left(M_{0}\right)$, the above analysis yield that the meromorphic function $y v f_{3}: M_{0} \rightarrow \mathbb{C}$ takes the same values on the points of $\tilde{l}$ which are symmetric with respect to $S_{h}$. Furthermore, as $S_{h}$ has fixed points, $u \circ S_{h}=1 / \bar{u}$, and so the 
function $R=y v h_{3}$ takes the same values on the points of $\tilde{l}$ which are symmetric with respect to $S_{h}$ too.

Summarizing, we may define in a natural way a meromorphic function $R(y)$ in the $y$-plane $\mathbb{C}^{*}$, in such a way that

$$
\phi_{3}^{0}=\frac{R(y)}{\sqrt{y\left(y-e^{-n b_{0} i}\right)\left(y-e^{n\left(t_{0}+\pi\right) i}\right)}} d y .
$$

Here, $\sqrt{y\left(y-e^{-n \omega_{0} i}\right)\left(y-e^{n\left(b_{0}+\pi\right) i}\right)}$ represents the well defined meromorphic function on $M_{0}$ given by $v u^{p}$.

Case 2: ord $\left(J_{0}\right)=\infty$. In this case, $n \in[1,2[-\mathbb{Q}$.

Let $\tilde{\mathscr{S}}$ be the covering Riemann surface of $\mathscr{S}$ where the function $u=\log (y)$ is well defined and label

$$
\mathfrak{p}: \tilde{\mathscr{S}} \rightarrow \mathscr{S}
$$

as the natural projection. The map $\mathfrak{p}$ is an infinitely many sheeted cyclic covering, and its group of deck transformations is generated by

$$
\tilde{J}_{0}((y, v), u)=((y, v), u+2 \pi i) .
$$

Defining $\mathscr{M}_{0}=\mathfrak{p}^{-1}\left(\Lambda_{0}\right)$ and reasoning as in the 1-st case, we infer that the cyclic covering

$$
\left.\mathfrak{p}\right|_{\mathscr{M}_{0}}: \mathscr{M}_{0} \rightarrow \Lambda_{0}
$$

is conformally equivalent to $\xi$.

Up to this biholomorphism, we may assume $\mathscr{M}_{0}=M_{0}$ and $\widetilde{J}_{0}=J_{0}$. The functions $y$ and $v$, up to composition with the covering map $\mathfrak{p}$, are meromorphic functions on $M_{0}$. Taking into account that $y=g_{0}^{n}$, we deduce also that, up to a suitable choice of the initial condition, $g_{0}=e^{u / n}$.

As in the 1-st case, $\left(\phi_{1}^{0}, \phi_{2}^{0}, \phi_{3}^{0}\right)$ are the holomorphic 1-forms of the Weierstrass data of $X_{0}$.

It is obvious that $J_{0}^{*}\left(\phi_{3}^{0}\right)=-\phi_{3}^{0}$, and so the meromorphic function $f_{3}=\phi_{3}^{0} / d y$ satisfies $f_{3} \circ J_{0}=-f_{3}$. This implies that $f_{3}=e^{u / 2} h_{3}$, where $h_{3}$ is the lift to $M_{0}$ of a meromorphic function on $\Lambda_{0}$.

Reasoning as in the 1-st case, we have $y \circ S_{h}=1 / \bar{y}, v \circ S_{h}=e^{n \pi i / 2} \bar{v} / \bar{y}, u \circ S_{h}=-\bar{u}$. Hence $h_{3}=R(y) /(y v)$, where $R(y)$ is a meromorphic function in the $y$-plane $\mathbb{C}^{*}$, and so

$$
\phi_{3}^{0}=\frac{R(y)}{\sqrt{y\left(y-e^{-n t_{0} i}\right)\left(y-e^{n\left(b_{0}+\pi\right) i}\right)}} d y
$$


where $\sqrt{y\left(y-e^{-n t_{0} i}\right)\left(y-e^{n\left(i_{0}+\pi\right) i}\right)}$ is the well-defined function on $M_{0}$ defined by $v e^{u / 2}$.

In both cases, we have concluded that

$$
y=g_{0}^{n}, \quad \phi_{3}^{0}=\frac{R(y)}{\sqrt{y\left(y-e^{-n t_{0} i}\right)\left(y-e^{n\left(t_{0}+\pi\right) i}\right)}} d y
$$

but $M_{0}$ is different in 1-st and 2-nd cases.

However, and in both cases, the function $R(y): \mathbb{C}^{*} \rightarrow \mathbb{C}$ is constant. Indeed, if we consider $D^{\prime}=\zeta^{-1}(D)$, where $D$ is a small enough neighbourhood of either 0 or $\infty$, then any connected subset $D^{\prime \prime}$ of $M^{\prime}$ whose projection onto $M_{0}$ is $D^{\prime}$ can be identified in a natural way with $D^{\prime}$. Hence, the immersion $\left.X_{0}\right|_{D^{\prime}}$ can be identified with $\left.X^{\prime}\right|_{D^{\prime \prime}}$. Furthermore, Theorem 3.12 yields that the third coordinate function

$$
X_{3}^{\prime}=\operatorname{Re} \int \frac{R(y)}{\sqrt{y\left(y-e^{-n k_{0} i}\right)\left(y-e^{n\left(l_{0}+\pi\right) i}\right)}} d y
$$

of $X^{\prime}$ is bounded at $D^{\prime \prime}$. The Great Picard Theorem implies easily that $R(y)$ has no essential singularities at 0 and $\infty$, and thus, $R(y)$ is a quotient of polynomial functions. As $g^{\prime}$ has no zeroes or poles in $M^{\prime}$ (see Lemma 3.22), the same holds for $g_{0}$, and so we deduce that $R(y)=C y^{k}, C \in \mathbb{C}^{*}, k \in \mathbb{Z}$. Using once again that $X_{3}$ is bounded around any end, we deduce that $k=0$, and thus $R(y)=C$ is constant.

Taking into account that $\sqrt{y} \circ S_{h}=1 / \overline{\sqrt{y}}, v \circ S_{h}=e^{n \pi i / 2} \bar{v} / \bar{y}$ and $S_{h}^{*}\left(\phi_{3}^{0}\right)=-\overline{\phi_{3}^{0}}$, it is not hard to check that, up to rigid motions,

$$
C=\frac{B}{n} e^{n \pi i / 4}, \quad B \in \mathbb{R}^{*} .
$$

Thus, (14) becomes:

$$
y=g_{0}^{n}, \quad \phi_{3}^{0}=\frac{B}{n} \frac{e^{n \pi i / 4}}{\sqrt{y\left(y-e^{-n t_{0} i}\right)\left(y-e^{n\left(t_{0}+\pi\right) i}\right)}} d y
$$

Consider the Riemann surface $\left\{(x, v) \in \mathbb{C}^{*} \times \mathbb{C} / \nu^{2}=\left(x+e^{t_{0} i}\right)\left(x-e^{-t_{0} i}\right)\right\}$, and define $\Lambda_{1}$ as the closure of one of the two connected components of $x^{-1}(\Lambda)$ (see Figure 12).

From Lemma 3.31, it is straightforward to check that, for a suitable choice of the branch of $\sqrt{\left(x+e^{t_{0} i}\right)\left(x-e^{-t_{0} i}\right)}$, the map

$$
F: M \rightarrow \Lambda_{1}, \quad F(P)=\left(g(P), \sqrt{\left(g(P)+e^{t_{0} i}\right)\left(g(P)-e^{-t_{0} i}\right)}\right)
$$

is a conformal diffeomorphism. So, $\Lambda_{1}$ and the holomorphic 1-forms $\phi_{j}=\left(F^{-1}\right)^{*}$ $\left(\left.\phi_{j}^{0}\right|_{M}\right), j=1,2,3$ are Weierstrass data for the immersion $X$.

Therefore, up to this biholomorphism, we have

$$
M \equiv \Lambda_{1}, \quad g=x, \quad \phi_{3}=B \frac{e^{n \pi i / 4} x^{n-1}}{\sqrt{x^{n}\left(x^{n}-e^{-n t_{0} i}\right)\left(x^{n}-e^{n\left(b_{0}+\pi\right) i}\right)}} d x,
$$


where the branch of $x^{n}$ is determined by the initial condition $1^{n}=1$.

After the change $z=-i x, M$ becomes the closure of $z^{-1}(-i \Lambda)$ in the Riemann surface $\mathscr{N}=\left\{(z, w) \in \mathbb{C}^{*} \times \mathbb{C} / w^{2}=\left(z-e^{i x_{0} / n}\right)\left(z-e^{-i x_{0} / n}\right)\right\}$, where $x_{0}=n\left(t_{0}+\pi / 2\right)$. Since $\left.t_{0} \in\right]-\pi / 2, \theta / 2\left[\right.$, then (13) gives $\left.x_{0} \in\right] 0, \pi[$.

Up to this change, the Weierstrass data of $X: M \rightarrow \mathbb{R}^{3}$ is given by:

$$
M=z^{-1}(-i \Lambda), \quad g=i z, \quad \phi_{3}=B \frac{d z}{z \sqrt{z^{n}+z^{-n}+2 r}} .
$$

where $r=-\cos \left(x_{0}\right)$.

These are the Weierstrass data exhibited in Section 2.

As we have assumed that the distance between the planes $\pi_{1}$ and $\pi_{2}$ is 1 , the value of $B$ is uniquely determined.

Moreover, Theorem 2.5 says that the immersion $X: M \rightarrow \mathbb{R}^{3}$ is, up to a horizontal translation, one of the surfaces $X_{\theta r}$ described in (7), where $\left.r \in\right]-1, r_{\theta}[$. This concludes the proof.

\section{References}

[1] A. D. Alexandrov, 'Uniqueness theorems for surfaces in the large I', Vestnik Leningrad. Univ. 11 (1956), 5-17; English translation: Amer. Math. Soc. Transl. (2) 21 (1962), 341-354.

[2] R. B. Burckel, An introduction to classical complex analysis vol. 1 (Birkhäuser, Basel, 1979).

[3] U. Dierkes, S. Hildebrandt, A. Küster and O. Wohlrab, Minimal Surfaces I, Grundlehren Math. Wiss. 295 (Springer, Berlin, 1992).

[4] Y. Fang, 'Minimal annuli in $\mathbb{R}^{3}$ bounded by non-compact complete convex curves in parallel planes', J. Austral. Math. Soc. (Series A) 60 (1996), 369-388.

[5] D. Hoffman and W. H. Meeks III, 'The asymptotic behavior of properly embedded minimal surfaces of finite topology', J. Amer. Math. Soc. (4) 2 (1989), 667-682.

[6] H. Jenkins and J. Serrin, 'Variational problems of minimal surface type. Il. Boundary value problems for the minimal surface equation', Arch. Rat. Mech. Anal. 21 (1966), 321-342.

[7] H. Karcher, 'Construction of minimal surfaces', in: Surveys in Geometry $1989 / 90$ (University of Tokyo, Tokyo, 1989). Also in: Vorlesungsreihe Nr. 1, SFB 256, Bonn, 1989.

[8] F. J. López, R. López and R. Souam, 'Maximal surfaces of Riemann type in Lorentz-Minkowski space $\mathbb{L}^{3}$, Michigan Math. J., to appear.

[9] F. J. López and F. Martín, 'Minimal surfaces in a wedge of a slab', Comm. Anal. Geom., to appear.

[10] F. J. López and F. Wei, 'Properly immersed minimal discs bounded by straight lines', Math. Ann., to appear.

[11] W. H. Meeks III and H. Rosenberg, 'The geometry and conformal structure of properly embedded minimal surfaces of finite topology in $\mathbb{R}^{3}$, Invent. Math. 114 (1993), 625-639.

[12] J. C. C. Nitsche, 'A supplement to the condition of J. Douglas', Rend. Circ. Mat. Palermo (2) 13 (1964), 192-198.

[13] - Lectures on minimal surfaces, vol. 1 (Cambridge University Press, Cambridge, 1989).

[14] R. Osserman, A survey of minimal surfaces, 2nd edition (Dover Publications, New York, 1986). 
[15] W. Rossman, 'Minimal surfaces with planar boundary curves', Kyushu J. Math. 52 (1998), 209225.

[16] R. Schoen, 'Uniqueness, symmetry and embeddedness of minimal surfaces', J. Differential Geom. 18 (1983), 791-809.

Departamento de Geometría y Topología

Universidad de Granada

\section{Granada}

Spain

e-mail: fjlopez@ugr.es,fmartin@ugr.es 